\title{
Freedom to act enhances the Sense of Agency, while movement and goal-related prediction errors reduce it
}

33

4

54

6

75

8

107

118

12

139

1410

15

1611

1712

18

1913

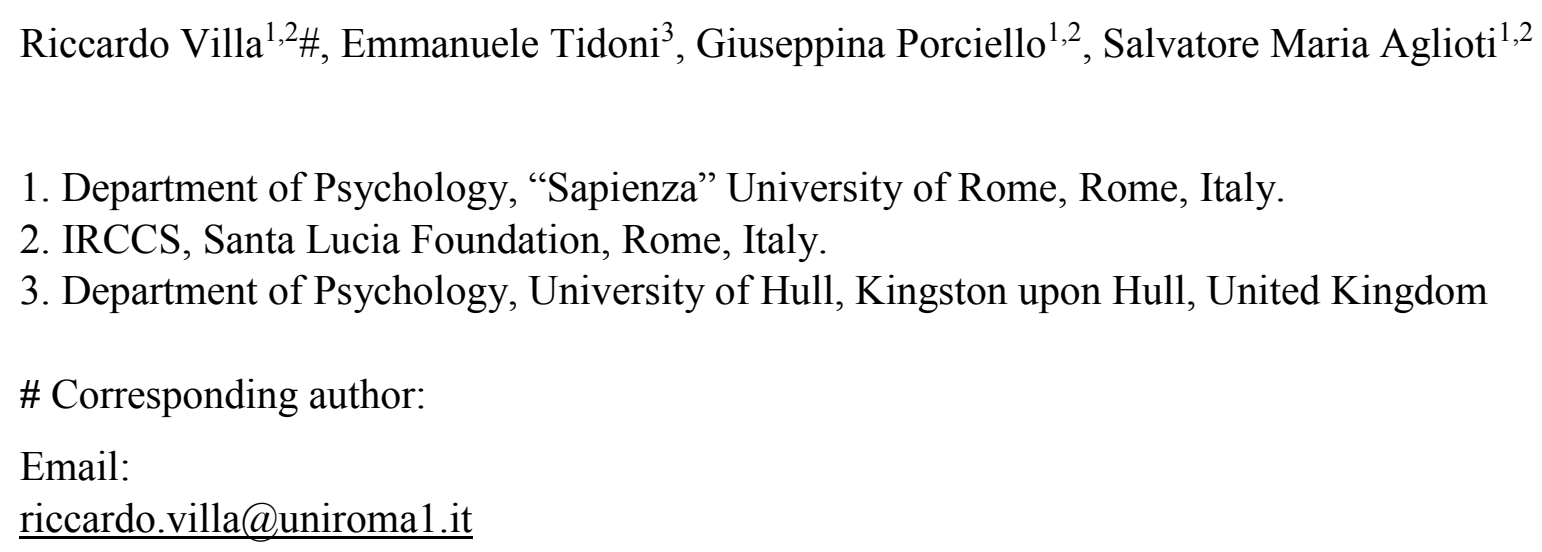

\section{Acknowledgements}

The study was supported by PRIN grant (Progetti di Ricerca di Rilevante Interesse Nazionale, Edit. 2015, Prot. 20159CZFJK; and Edit. 2017, Prot. 2017N7WCLP) and by European Research Council (ERC) Advanced Grant (eHONESTY, Prot. 789058) to SMA. 


\section{ABSTRACT}

The Sense of Agency (SoA) is the experience of controlling one's movements and their external consequences. Accumulating evidence suggests that freedom to act enhances SoA, while prediction errors are known to reduce it. Here, we investigated if prediction errors related to movement or to the achievement of the goal of the action exert the same influence on SoA during free and cued actions. Participants pressed a freely chosen or cued color button, while observing a virtual hand moving in the same or in the opposite direction - i.e., movement-related prediction error - and pressing the selected or a different color - i.e., goal-related prediction error. To investigate implicit and explicit components of SoA, we collected indirect (i.e., Synchrony Judgments) and direct (i.e., Judgments of Causation) measures. We found that participants judged virtual actions as more synchronous when they were free to act. Additionally, movement-related prediction errors reduced both perceived synchrony and judgments of causation, while goal-related prediction errors impaired exclusively the latter. Our results suggest that freedom to act enhances SoA and that movement and goal-related prediction errors lead to an equivalent reduction of SoA in free and cued actions. Our results also show that the influence of freedom to act and goal achievement may be limited respectively to implicit and explicit SoA, while movement information may affect both components. These findings provide support to recent theories that view SoA as a multifaceted construct, by showing that different action cues may uniquely influence the feeling of control.

\section{Keywords}

Sense of Agency, Movement, Goal, Free Choice, Prediction errors, Action Monitoring, Behavioral Adjustments, Virtual Scenario 


\section{INTRODUCTION}

The Sense of Agency (SoA) is the experience of controlling one's movements and their consequences in the external environment (Aarts et al., 2012; Moore \& Fletcher, 2012; Tsakiris, Longo, \& Haggard, 2010). Recent theoretical models suggest that SoA is a multi-faceted experience that comprises both implicit and explicit components (Moore \& Fletcher, 2012; Synofzik, Vosgerau, \& Newen, 2008a, 2008b; Synofzik, Vosgerau, \& Voss, 2013; Wegner \& Sparrow, 2004). Specifically, it has been proposed that SoA involves an implicit, non-conceptual component - i.e., feeling of agency - that relies mostly on sensorimotor information, and an explicit, conceptual and interpretative component - i.e.,judgment of agency - that relies on the formation of beliefs about the causes of actions and their consequences (Synofzik et al., 2008a, 2008b). This distinction implies that SoA may depend on a set of multiple cues, such as contextual information and a comparison between the predicted and actual consequences of actions (Moore \& Fletcher, 2012; Synofzik et al., 2013). This work focuses on the contribution to SoA of different prediction errors - movement and goal-related - within free and cued contexts of action.

Previous research consistently reported that prediction errors influence SoA. Prediction errors can broadly be defined as a mismatch between prior expectations and reality (den Ouden, Kok, \& de Lange, 2012). Within the "active inference" framework of perception and action (Friston, 2010, 2012; Friston, Samothrakis, \& Montague, 2012), prediction errors can be considered as the discrepancy between the bottom-up sensory input and the top-down predictions made by the brain about that input (Friston, 2011; Wolpe \& Rowe, 2014a). Prediction errors are thought to play a central role in motor control, by signaling the difference between the predicted and the actual outcome of the action (Haggard, 2017; Wolpe \& Rowe, 2014b). Interestingly, a recent study revealed that prediction errors also occur in a dyadic motor interaction when a virtual partner performs an unexpected action., i.e. violating the initial prediction (Moreau, Candidi, Era, Tieri, \& Aglioti, 2019). Indeed, it is generally assumed that the brain forms predictions about how the action will unfold and about its sensory consequences (Blakemore \& Frith, 2003; Blakemore \& Sirigu, 2003; Wolpert, Ghahramani, \& Jordan, 1995). Predictions are then compared with the actual events. If they match, no prediction error is generated, conversely, error signals are generated. According to one of the most popular theories of SoA - the Comparator Model (Blakemore, Wolpert, \& Frith, 2002; Frith, Blakemore, \& Wolpert, 2000) - prediction errors contribute both to adjust behavior and to modulate SoA. In particular, SoA would be experienced in the absence of prediction errors, and it would be reduced by their occurrence. Indeed, SoA was found to be reduced by movement-related prediction errors - i.e., by the observation of unpredicted movements that do not correspond to the ones executed by the participants (Daprati et al., 1997; David, Skoruppa, Gulberti, Schultz, \& Engel, 2016; Farrer et al., 2008; van den Bos \& 
Jeannerod, 2002) - and by outcome-related prediction errors - i.e., by the occurrence of unexpected outcomes following participant's action (Caspar, Desantis, Dienes, Cleeremans, \& Haggard, 2016; David et al., 2016; Kühn et al., 2011; Sato \& Yasuda, 2005).

However, the idea that prediction errors systematically reduce SoA has been criticized by Synofzik and colleagues (Synofzik et al., 2008a), who observed that the comparator model fails to explain situations where people experience SoA despite small prediction errors, or the finding that individuals may experience a "vicarious" SoA for observed actions even in absence of real movements (Tieri, Tidoni, Pavone, \& Aglioti, 2015; Wegner, Sparrow, \& Winerman, 2004). Similarly, the comparator model may fail to account for the influence on SoA of low level bodily signals, such as breath (Monti, Porciello, Tieri, \& Aglioti, 2020). Hence, SoA may depend on many sources of information, which would include - but would not be limited to - prediction errors. In a previous study (Villa, Tidoni, Porciello, \& Aglioti, 2018) we tested the influence on SoA of three types of prediction errors, namely, prediction errors about i) movement execution, ii) goal achievement, and iii) the time in which these events should occur. We devised a novel paradigm, the SoA-GAME - i.e., Sense of Agency for Goal Achievement and Movement Execution - in which participants performed simple goal-directed actions. Their task was to press a button of a cued color and to observe a virtual hand performing the same or a different action from a first-person perspective. Specifically, the virtual finger could move in the same or in the opposite direction with respect to the participants' finger and the color pressed in the virtual scenario could be the one selected by the participants or a different one. Additionally, delays of increasing duration were introduced between the executed and virtual action. By orthogonally manipulating movement (same/opposite), goal (achieved/missed) and time information (synchronous/delayed), we were able to measure the unique contribution of these cues to SoA. Tellingly, our data indicated that both movement and goal-related prediction errors reduce SoA, but also that movement information seems to be a more constant source of SoA modulation than goal information.

It is important to underline that in our previous version of the task the goal of participant's action was defined by an external cue. Hence, it is unclear whether the same effects could be observed also for freely chosen actions.

Being internally generated and independent from environmental influences (Fried, Haggard, He, \& Schurger, 2017; Haggard, 2017) voluntary actions have been often identified by contrasting them to reflexes and to actions guided by external cues, i.e., cued actions (Fried et al., 2017; Frith, 2013). Voluntariness is often viewed as a fundamental cue to SoA (Haggard, 2017) and indeed accumulating evidence suggests that freedom to act enhances SoA.

For instance, Wenke and colleagues asked their participants to freely press one of two buttons to 
obtain a visual outcome on screen (Wenke, Fleming, \& Haggard, 2010) or to press one of two buttons following an instruction. Participants reported higher control over the outcome when they were free to act as compared to when they followed an instruction. In a series of studies, Barlas and colleagues (Barlas, Hockley, \& Obhi, 2017, 2018; Barlas \& Obhi, 2013) compared intentional binding between freely chosen and cued actions. Intentional binding (Haggard, Clark, \& Kalogeras, 2002; Moore \& Obhi, 2012) is a perceived time compression between the action and its outcome. Although recent evidence suggests that intentional binding may simply reflect multisensory integration associated to causal inference (Kirsch, Kunde, \& Herbort, 2019; Poonian \& Cunnington, 2013; Suzuki, Lush, Seth, \& Roseboom, 2019), intentional binding was classically considered an implicit marker of SoA. Participants reported stronger binding when they were free to decide which action to perform, suggesting that freedom to act was associated to higher implicit SoA. The same trend was observed when participants provided explicit judgments of agency (Barlas et al., 2017, 2018).

In line with these findings, recent studies show that SoA is significantly reduced when participants are coerced to perform an action. In a series of experiments, Caspar and colleagues asked participants to perform button presses that could result either in an mildly painful electrical shock delivered to a co-participant or in taking part of his/her remuneration (Caspar, Christensen, Cleeremans, \& Haggard, 2016; Caspar, Cleeremans, \& Haggard, 2018; Caspar, Vuillaume, Magalhães De Saldanha da Gama, $\&$ Cleeremans, 2017). Simultaneously to the harmful outcome participants heard a tone and were asked to estimate the time interval between action and tone and thus provide a measure of intentional binding. Importantly, actions could be executed in a context of freedom of choice, or upon a specific request by the experimenter (i.e., coercive condition). Intentional binding was significantly reduced when participants were coerced to perform an action as compared to the freedom of choice context. Together, these studies bring additional evidence that freedom to act is crucial for SoA. However, previous studies did not systematically investigate if the enhancement of SoA generated by freedom to act is also linked to changes of the impact on SoA of failures to control one's own movements or to achieve the goal of the action. In other words, it is not clear whether movement and goal-related prediction errors are equally effective in reducing SoA within free and cued contexts of action. In a previous study, Barlas and Kopp (2018) reported that SoA - measured by means of intentional binding and of explicit reports of their feeling of control - was independently reduced when participants freedom to act was limited and when incongruent outcomes occurred. However, Barlas and Kopp study did not allow a direct comparison of movement and goal related prediction errors, since their task only included the presentation to participants of incongruent outcomes. Here, we adapted our SoA-GAME paradigm so that participants could perform actions both in contexts of freedom of choice and following imperative cues. This way, we sought to directly compare the effects 
of movement, goal and time-related prediction errors when the individual performs free or cued actions. As mentioned earlier, SoA may include implicit and explicit components. To capture the effects of our manipulations on these two components of SoA we employed two measures. In the first session of the experiment participants were asked to judge if the observed virtual action took place simultaneously to their action or with a delay. This measure, referred to as synchrony judgments (Villa et al., 2018) allowed us to collect information about participants' implicit SoA. Indeed, a previous study suggested that synchrony judgments rely on the same source of information involved in expressing agency judgments (Weiss, Tsakiris, Haggard, \& Schütz-Bosbach, 2014). This conclusion was based on fMRI data showing that the inferior parietal cortex was activated both when participants noticed delays between their action and visual feedback of their action and when participants reported a reduction of SoA by attributing the observed action to someone else (Farrer et al., 2008). Consistently, a recent study found that the ability to detect delays is negatively correlated with explicit agency ratings - the better participants were at detecting delays between action and outcome, the less they reported to feel in control when delays occurred (Osumi et al., 2019). Hence, previous evidence suggested that Synchrony Judgments may allow to measure implicit SoA. In the second session of the experiment we collected a direct measure of explicit SoA by asking participants to rate their feeling of causing the virtual actions by means of a Visual Analogue Scale (VAS) (similarly to Pezzetta et al., 2018). This measure was adapted from previous studies that investigated explicit SoA over the movements of a virtual (Salomon et al., 2016; Tieri et al., 2015), robotic (Caspar, Cleeremans, \& Haggard, 2015) or rubber hand (Braun, Thorne, Hildebrandt, \& Debener, 2014; Kalckert \& Ehrsson, 2012, 2014). By adding this measure, which was not present in our previous version of the task (Villa et al., 2018), we sought to capture the similarities and differences between the effects induced by our manipulations respectively on implicit and explicit SoA. We hypothesized that freely chosen actions would be associated with higher SoA (i.e., higher perceived synchrony/higher judgments of causation) as compared to cued actions and that participants would experience a decrease of SoA (i.e., lower perceived synchrony/lower judgments of causation) after observing both movement and goal-related prediction errors similarly to our previous findings (Villa et al., 2018). However, in line with recent evidence (Beck, Di Costa, \& Haggard, 2017; Borhani, Beck, \& Haggard, 2017) we also expected that information about achievement of the goal of the action would influence SoA more in free than in cued actions: we expected that participants report higher synchrony/judgments of causation when they achieve a freely chosen goal as compared to a cued goal, and that a failure to achieve the goal may lead to a sharper reduction of the reported synchrony/judgments of causation in the free than in the cued context of action. However, we also expected differences between implicit and explicit measures of SoA: given that individuals tend to 
self-attribute successful outcomes (Arkin, Appelman, \& Burger, 1980; Miller \& Ross, 1975), information about goal achievement may affect more SoA at an explicit level. Finally, considering that SoA and motor performance may both be influenced by the same type of prediction errors (Haggard, 2017), we tested whether our manipulations could also induce "post-error adjustments". Making an error is known to affect performance in following trials, which is generally referred to as post-error adjustments (Danielmeier \& Ullsperger, 2011; Fusco et al., 2018; Ullsperger, Danielmeier, \& Jocham, 2014). For instance, participants perform actions more slowly in trials following an error, an effect known as Post-Error Slowing (PES). Interestingly, post-error adjustments were also reported after prediction errors and unexpected visual consequences of actions (Gentsch, Ullsperger, \& Ullsperger, 2009; Padrao, Gonzalez-Franco, Sanchez-Vives, Slater, \& Rodriguez-Fornells, 2016; Wessel \& Aron, 2013; Wessel, Danielmeier, Morton, \& Ullsperger, 2012). We measured behavioral adjustments by calculating the amount of time participants took to a) provide synchrony judgments i.e., Judgment Times - and b) perform a new action after observation of every type of virtual action - i.e., post observation Reaction Times. We expected that movement and goal-related prediction errors would be associated to increased Judgment Times and post observation Reaction Times. In principle, one possibility was that our manipulations would generate the same effects on behavioral adjustments and on SoA. However, in light of recent theories that suggest that the magnitude of error signals may not necessarily translate in an equivalent reduction of SoA (Synofzik et al., 2008a, 2008b), movement and goal-related prediction errors would have a different impact on behavioral adjustments and SoA, respectively.

\section{MATERIALS AND METHODS}

\subsection{Participants}

To estimate the sample size, we performed a power analysis (MorePower 6.0.4, Campbell \& Thompson, 2012). We used the effect size of the significant interaction between factors Goal and Delay, i.e., $\eta_{p}{ }^{2}$ of 0.123 , reported in our previous analysis of Synchrony Judgments where we employed the SoA-GAME (Villa et al., 2018). We chose to focus on this measure because Synchrony Judgments was the only measure of SoA that we employed in our previous study. Moreover, we chose this effect size because i) the interaction between factors Goal and Delay was the highest-order statistically significant interaction; and that ii) this interaction showed the smallest effect size among the significant effects observed in our previous data making maximally conservative our choice. Thus, to achieve a power of 0.80 for this effect and considering the $2 \times 2 \times 2 \times 3$ design (see below) of our study, the estimated sample size was 36 . Moreover, to fully counterbalance our experimental design 
(see the section 2.3 Procedure and Task) we decided to collect data from 45 participants. All participants were right-handed, had no prior history of neurological or psychiatric disorders, had normal or corrected to normal visual acuity and were not color-blind. Participants were naïve with respect to the purposes of the study and explanations of the hypotheses were provided at the end of the experiment. The experimental protocol was approved by the ethics committee of Fondazione Santa Lucia (Prot. CE/PROG. 686) and was performed in accordance with the 1964 declaration of Helsinki. All participants read and provided written informed consent to take part in the study. Five participants were excluded from the final sample and from the analyses since they failed to meet predefined exclusion criteria (for details see the "Excluded Participants" section of the supplementary materials). The final sample was thus composed of 40 participants (20 males; age range: 19-31 years; mean \pm S.E.M: $23.7 \pm 0.408)$.

\subsection{Apparatus}

A Matlab (The MathWorks, Inc) custom script was used to run the experiment. A virtual scenario (Fig. 1) created by means of 3DS Max 2011 (Autodesk, Inc) was shown on a monitor (Benq GL $2250-\mathrm{T}$; refresh rate, $60 \mathrm{~Hz}$; resolution set to $1280 \times 720$ pixels). This included a virtual humanoid right-limb (forearm and hand) and a virtual response box, composed of two dark grey buttons respectively attached to the top and to the bottom of a transparent structure. The index of the virtual hand laid between the two buttons of the virtual response box.

The monitor was sustained by a wooden structure located on the table, so that it was inclined of $12.7^{\circ}$ with respect to the horizontal plane. Participants inserted their right arm in a rectangular hole at the front of the structure $(58 \times 8.5 \mathrm{~cm})$. They were asked to lay their arm on the table in order to match the position of the virtual arm. The presence of the screen prevented participants to observe their real arm and a piece of black cloth was used to cover their shoulders and the elbow joints to prevent any visual discontinuity between the virtual limb and participants' body. A custom-made response box, closely matching the features of the virtual response box, was placed on the table below the monitor. The response box was C-shaped and included two identical USB numeric pads. The keys of the two devices faced each other's. The upper pad was sustained at a height of $7 \mathrm{~cm}$ by a plastic support fixed on the table. Two plastic buttons (height: $1.5 \mathrm{~cm}$ ), with a squared and flat top face (side length: 3.2 $\mathrm{cm})$ were attached respectively to the " 2 " and " 5 " keys of the lower and upper pads and allowed to record downward and upward movements of the index finger. Before starting the experiment, the distance between the surfaces of the two plastic buttons was adapted for each participant by inserting paper supports below the lower pad, so that the dorsal part of the distant phalanx of the index touched the superior button, while the ventral part rested on the inferior button, and the two plastic buttons were vertically aligned. Finally, a standard USB keyboard was placed to the left of the monitor and 
allowed participants to answer to the specific question that appeared on screen at the end of a trial (See "Procedure and task" and Fig. 1 for details).

\section{Please insert Fig. 1 about here}

\subsection{Procedure and task}

The experiment was performed in a dimly lit room. Participants sat comfortably on a chair, at a viewing distance of approximately $40 \mathrm{~cm}$ from the center of the screen. During the experiment, the two virtual buttons turned to yellow and blue, respectively. Participants' task was to select one of the two virtual buttons according to its color and to press the real button in the corresponding position as fast as possible, by performing an upward or downward movement. In separate blocks, participants could either freely select which color to press - i.e., Free Block - or select the color according to an imperative cue - i.e., Cued Block (see below for details). Pressing the real button triggered the observation of an action (visual feedback) in the virtual scenario. The virtual action could be similar or different from the one performed by the participant and it took place as soon as a button press was detected or with different possible delays (see the section 2.4 ActionOutcome manipulation for details). Then, an indirect - Synchrony Judgments, SJs - or direct Judgments of Causation, JoC - measure of SoA was collected (see below and Fig. 2, panel A for details). These two measures were collected separately in two different sessions of the experiment the SJ session and JoC Session - each composed of a Free and Cued block. The order of Free and Cued blocks within the two sessions was counterbalanced across participants, while the two sessions followed a fixed order: participants always performed the SJ session before the JoC session (see Fig. 2, panel A). By keeping the order of SJ and JoC sessions fixed, we aimed at controlling potential response biases that could have been induced in the expression of synchrony judgments by the prior exposure to the explicit measure of SoA (for a similar approach, see Braun et al., 2014).

Specifically, in the SJ session participants provided SJs by judging as fast as possible if the observed visual "change in the virtual scenario" took place simultaneously to their action or delayed. They were informed that by "change in the virtual scenario" we referred to the fact that contingently on their button press, they would observe the virtual index pressing a virtual button of a certain color. We considered that using this terminology - instead of "observed action" - would not bias participants to focus on the movement of the virtual finger over the color of the pressed virtual button. Participants were asked to respond to the question by only focusing on the temporal contiguity 
between their action and the visual change in the virtual scenario, irrespective of the type of observed action. Two keys of a standard USB keyboard were respectively labeled "S" for Synchronous and "A" for Asynchronous, and participants used their left index and the middle fingers to respond. The finger (Index/Middle) used to respond "Synchronous" was fully counterbalanced across participants. Additionally, to check that participants were aware of the disposition of the colors when performing a button press, we added a control question. In a sub-set of trials participants were asked to report if "the final disposition of the colors - observed following the virtual action - was reversed with respect to the initial one - observed before performing the action" (see the section 2.4 Action-Outcome Manipulation - for more details). To answer to this question participants used the same fingers and keys as for SJs. They pressed S for "Yes" (the final disposition was reversed with respect to the initial one) and A for "No" (the disposition of the two colors did not change).

In the JoC session participants expressed judgments of causation. After each virtual action observation, they were asked to rate how much they felt they had caused the visual change in the virtual scenario. Participants were informed that they could choose between all the values of a 100 points VAS spanning from 0 ("Not at all") to 100 ("Completely") and to press a third key, labelled "enter", to confirm their answer. As for SJs, we referred to the visual change to avoid that participants would focus on the movement of the virtual finger over the color of the pressed virtual button.

\subsubsection{Cued Block}

The structure of a typical trial in the Cued block was as follows. A tone signaled the beginning of the trial, and at the same time a blue or yellow circle (i.e., a cue) appeared at the left of the virtual response box, at the same height of the virtual index and at equal distance from the two virtual buttons. The color of the circle instructed participants about which real button they should press in the current trial - i.e., if yellow, they had to press the real button that was in the same position of the virtual button that turned to yellow; if blue, they had to press the real button that was in the same position of the virtual button that turned to blue. The color of the circle was random for each trial and participants observed an equal number of times the two types of cues. The circle remained visible for $1000 \mathrm{~ms}$ and then disappeared. After a random time (included between 1000 and $1500 \mathrm{~ms}$ ) the two virtual buttons flashed, one of them turning blue and the other yellow, for $120 \mathrm{~ms}$. The two possible dispositions of the colors (yellow up, blue down and vice-versa) were presented an equal number of times, and their order of presentation was randomized for each participant. The colors of the two virtual buttons then returned dark grey, and participants had to press the real button corresponding to the position of the cued color with an upward or downward movement. If no response was provided within three seconds the current trial was aborted. Moreover, if the participant pressed the wrong 
button (e.g., the cued color appeared above the virtual index finger, but the participant pressed the lower real button) a "prohibition sign" was displayed for $2000 \mathrm{~ms}$ and the trial was aborted. Aborted trials were repeated at the end of the block. When participants pressed the cued button, a visual feedback simultaneous or delayed with respect to the button press was provided (see the section 2.4 Action-Outcome Manipulation and Fig. 2, panel C). The visual feedback remained visible for 500 ms. Then, the virtual hand and the virtual response box were covered by a black (for SJs and JoC) or grey (for control questions) rectangle and participants were asked to respond to the current question. The inter-trial interval (ITI) was set to $1000 \mathrm{~ms}$.

\subsubsection{Free Block}

The structure of a typical trial in the Free block was identical to the one described for the Cued block, with only one difference: in the Free block, the color of the circle appearing at the beginning of the trial was half yellow and half blue. The orientation of the circle (whether the left half was yellow or blue, See Fig. 2, panel B) was random for each trial and participants observed an equal number of times the two types of circles. This symbol was introduced to maintain a perceptual similarity with respect to the Cued block, and participants were asked to use it as a reminder that they should decide which color to press in that trial. The orientation of the virtual hand was identical with respect to the one employed in the Cued block. As in the Cued block, participants had to respond within three seconds, or the trial would be aborted. Participants were asked to: i) freely choose which color to press in each trial; ii) refrain from using a predefined strategy in choosing the color and iii) avoid pressing always the same color. Adherence to these constraints was assessed at the end of the experiment for each participant. After participants pressed the chosen button they were shown a visual feedback (see the section 2.4 Action-Outcome Manipulation and Fig. 2, panel C) and they were asked to respond to the $\mathrm{SJ} / \mathrm{J} / \mathrm{C}$ or control question.

\subsection{Action-Outcome Manipulation}

Pressing one of the two buttons of the response box triggered the observation of a visual feedback which could be simultaneous or delayed with respect to button press $(0 \mathrm{~ms},+150 \mathrm{~ms},+300 \mathrm{~ms})$. Indeed, the virtual finger could move in the same $(\mathrm{M}+)$ or in the opposite $(\mathrm{M}-)$ direction with respect to participant's movement and the goal could be achieved $\left(\mathrm{G}^{+}\right)$or missed $\left(\mathrm{G}_{-}\right)$depending on whether the virtual hand pressed the selected or the other color. The combination of movement and goal manipulations resulted in four possible types of feedback: one was fully correct $(\mathrm{M}+\mathrm{G}+)$, while three were erroneous $(\mathrm{M}+\mathrm{G}-, \mathrm{M}-\mathrm{G}+$, M-G-; see Fig. 2, panel $\mathbf{C}$ for a graphical representation of the four types of feedback).

To familiarize with the different types of feedback and with freely chosen and cued actions, 
participants performed practice trials before starting each of the two blocks that composed the SJ session. In these trials, the first 2 button presses were always followed by the observation of simultaneous $\mathrm{M}+\mathrm{G}+$ feedback; in twelve trials participants observed each of the possible Feedback $\mathrm{x}$ Delay combinations (e.g., $\mathrm{M}+\mathrm{G}+$, delay 0 , for a total of 12 possible combinations), and in 1 trial participants responded to the control question. Since participants could make errors, fail to perform an action within the given response window, or need to adjust the position of the hand to facilitate button presses, the overall number of trials during practice was not the same for all participants. They performed on average 15 trials (range: $15-17 ; \pm$ S.E.M.: 0.106) before starting the free block and 16 trials (range: $15-24 ; \pm$ S.E.M.: 0.277) before starting the free block. Data from practice trials were not included in the analysis.

In each session of the experiment ( $\mathrm{SJ} / \mathrm{JoC}$ ) and in each block (Free/Cued), the order of appearance of each Feedback x Delay combination was randomized. In the cued blocks, the color to press and the corresponding visual feedback were known before the participant performed the action. In the free blocks, the visual feedback was determined online according to the color chosen by the participant. To help participants familiarize with the experimental procedure, in each block the first 4 button presses were always followed by simultaneous $\mathrm{M}+\mathrm{G}+$ feedback. These trials were excluded from the analysis. In the SJ session each Feedback x Delay combination was presented 24 times, for a total of 288 trials (144 trials in each block, 12 trials per condition). Additionally, in 16 trials ( 8 per block, 2 per each type of feedback) participants were asked to respond to the control question aimed at assessing participants' awareness of the disposition of the colors. No delays between action and feedback were introduced when participants were required to respond to the control question. During the JoC session each Feedback x Delay combination was presented 8 times, for a total of 96 trials (48 trials in each block).

\section{Please insert Fig. 2 about here}

\subsection{Data Handling}

Although the number of trials for each Feedback $\mathrm{x}$ Delay combination was fixed, the total number of trials was not identical for each participant (for instance, participants could make errors in the cued blocks, or they could fail to perform an action within the given response window of 3 seconds - i.e., action failures). Participants performed on average 318 trials in the SJ session (Block: total trials range, Mean \pm S.E.M; Free block: 156-166, $157 \pm 0.274$; Cued block: 156-191, $161 \pm 1.022$ ), and 
106 trials in the JoC session (Free block: 52-53, $52 \pm 0.053$; Cued block: 52-61, $54 \pm 0.328$ ). We removed from the analysis i) real errors (in cued blocks) ii) action failures iii) trials where the experiment was suspended to adjust the position of participant's index finger to favor optimal reception of button presses i.e., interruptions (see table S2 in the supplementary materials for details). Finally, we analyzed participants' accuracy in responding to the control questions separately with respect to the rest of the trials (see paragraph 3.5).

After trial removal, analyses were performed on 288 trials per participant for the SJ session and on 96 trials for the JoC session.

We analyzed four dependent variables: three in the SJ sessions and one in the JoC sessions. For SJ trials, for each condition we calculated 1) the proportion of "Synchronous" answers to the synchrony judgments (i.e., SJs); 2) the mean amount of time participants took to provide an answer after observing a visual feedback in the virtual scenario (i.e., Judgment Times, JTs) and 3) the mean amount of time participants took to perform a new action in the trial that followed the observation of each specific type of feedback (post observation Reaction Times, poRTs). For JoC trials, we calculated the mean value representing the feeling of causing the virtual action for each condition (i.e., JoC). Mean values were calculated for each dependent variable, for each subject in each of the 24 conditions resulting from the combination of 4 independent variables: Context (Free/Cued), Movement (Congruent, Incongruent), Goal (Achieved/Missed) and Delay ( $+0 \mathrm{~ms},+150 \mathrm{~ms},+300 \mathrm{~ms})$. Before running parametrical statistical tests, we checked normality assumption by verifying that at least one of the following criteria was met (Field et al., 2013), namely that Kolmogorov-Smirnov test was not significant and that z-scores for skewness and kurtosis were included between -2.58 and +2.58 . No condition violated the abovementioned criteria in $\mathrm{JoC}$, while several conditions were not normally distributed for all dependent variables in the SJ session. To correct for this, SJs values were transformed by means of an ipsatization procedure (similarly to Tieri et al., 2015), an intra-subject standardization method that is carried out by subtracting the subject mean across conditions from the value obtained in a specific condition (Fischer, 2004; Fischer \& Milfont, 2010). Following the ipsatization procedure, positive scores indicate that the participant showed a higher perceived synchrony in that condition with respect to her/his mean, while negative scores indicate that the participant showed a lower perceived synchrony in that condition with respect to her/his mean. Hence, we calculated the mean reported synchrony for each subject across conditions, and we subtracted it from the individual values obtained in each condition (see Villa et al., 2018 for a similar appoach). After the ipsatization procedure 4 out of 24 conditions were still not normally distributed. Given the small number of conditions not meeting the normality assumption and the high number of conditions and of participants $(n=40)$, we decided to proceed with parametrical testing. However, to check the 
validity of our results on SJs, we also conducted a non-parametrical analysis that can be found in the Supplementary Materials. For JTs and poRTs we applied a square root transformation to the raw mean values so that no deviations from normality were found. SJs, JTs, poRTs and JoC data were entered into 4 separate $2 \times 2 \times 2 \times 3$ repeated measures Analysis of Variance (Anovas), with Context, Movement, Goal and Delay as within-subjects factors. The level of significance was set to .05 and Tukey correction was applied to all post-hoc comparisons. Statistical analyses were run using STATISTICA 8.

\section{RESULTS}

\subsection{Synchrony Judgments (SJs)}

The Anova on SJs revealed a main effect of factor Movement $\left(\mathrm{F}(1,39)=7.581, p=.009, \eta_{p}{ }^{2}=.163\right.$. Fig. 3, panel A). Participants perceived a congruent movement (mean \pm S.E.M., M+: $0.062 \pm 0.023$ ) as more synchronous than an incongruent movement (M-: -0.062 \pm 0.023$)$. Interestingly, the factor Context was also significant $\left(\mathrm{F}(1,39)=6.052 ; p=.018, \eta_{p}{ }^{2}=.134\right.$. Fig. 3, panel B): participants perceived the visual feedback as more synchronous in the free (Free: $0.017 \pm 0.007$ ) than in the cued block (Cued: $-0.017 \pm 0.007)$. The main effect of factor Delay was also significant $(\mathrm{F}(2,78)=74.830$, $p<.001, \eta_{p}{ }^{2}=.657$. Fig. 3, panel $\mathbf{C}$ ). This result confirms that participants correctly understood the meaning of the synchrony judgment question and that they could successfully discriminate increasing delays, which were all different from each other as confirmed by post-hoc comparisons (Delay(0): $0.250 \pm 0.029$; Delay(150): $0.015 \pm 0.016$; Delay(300): $-0.264 \pm 0.026$; all $p s<.001$; all $d s>1.608$ ) Importantly, the effects of factors Context and Delay were further explained by a Context x Delay interaction $\left(\mathrm{F}(2,78)=5.221, p=.007, \eta_{p}{ }^{2}=.118\right.$. Fig. 3, panel D). Post-hoc comparisons revealed that participants could discriminate increasing delays in both blocks (all $p s<.001$ and $d s>1.328$, see Table 1 for mean \pm S.E.M for each Context x Delay level). Importantly, feedback was perceived as more synchronous in the free block than in the cued block at Delay(150) $(p=.041, d=0.367)$ and $\operatorname{Delay}_{(300)}(p<.001 ; d=0.354)$. No difference between free and cued actions was observed when no delay was introduced after the button press $(p=.999)$. The Anova on SJs did not reveal any other significant main or interaction effects (all $F s<2.573$, all $p s>.083$ ).

\section{Please insert Fig. 3 about here}


6

\section{Please insert Table 1 about here}

\subsection{Judgments of Causation (JoC)}

The Anova on JoC revealed three main effects. Firstly, we found a main effect of factor Movement $\left(\mathrm{F}(1,390)=28.074, p<.001, \eta_{p}{ }^{2}=.419\right.$. Fig. 4, panel A). Participants expressed higher judgments of causation when the virtual finger moved in the same direction as the participant's one $(\mathrm{M}+: 53.070$ $\pm 2.942)$, as compared to the opposite one (M-: $35.907 \pm 2.971)$. Secondly, we found a main effect of factor Goal $\left(\mathrm{F}(1,39)=4.446, p=.041, \eta_{p}{ }^{2}=.102\right.$. Fig. 4, panel B). Participants expressed higher judgments of causation when the virtual hand pressed the virtual button of the selected color $(\mathrm{G}+$ : $45.928 \pm 2.592)$, as compared to the other one (G-: $43.048 \pm 2.539)$. Lastly, we found a main effect of factor Delay $\left(\mathrm{F}(2,78)=15.463, p<.001, \eta_{p}{ }^{2}=.284\right.$. Fig. 4, panel C). Participants reported higher judgments of causation for virtual feedback that immediately followed their actions as compared to delayed feedback. Post-hoc comparisons revealed that the three delays (Delay(0): $52.141 \pm 3.432$; $\operatorname{Delay}_{(150)}$ : $44.509 \pm 2.547$; Delay(300): $36.815 \pm 2.771$ ) were all significantly different (all $p s<.020$, all $d s>.399$ ), with lower judgments of causation for increasing delays. The Anova did not reveal any other significant main or interaction effects (all $F s<3.173$, all $p s>.083$ ).

\section{Please insert Fig. 4 about here}

\subsection{Judgment Times (JTs)}

The Anova on JTs revealed a main effect of factor Delay $\left(\mathrm{F}(2,78)=14.892, p<.001, \eta_{p}{ }^{2}=.276\right.$. Fig.5, panel A). Post-hoc analysis showed faster JTs when the delay between action and virtual feedback was of $300 \mathrm{~ms}$ (Delay(300): $0.828 \pm 0.016$ ), as compared to delays 0 (Delay(0): $0.865 \pm 0.017$. $p<.001, d=.351$ ) and 150 (Delay(150): $0.880 \pm 0.018, p<.001, d=.482$ ). Delays 0 and 150 did not significantly differ $(p=.119)$. A significant main effect of factor Goal was also found $(\mathrm{F}(1,39)=$ 30.218, $p<.001, \eta_{p}{ }^{2}=0.437$. Fig. 5, panel B). JTs were significantly slower after participants observed that the goal was missed (G-: $0.871 \pm 0.017)$, as compared to when it was achieved (G+: $0.845 \pm 0.016)$. Importantly, the effects of goal manipulation on JTs were further explained by a significant Movement x Goal interaction $\left(\mathrm{F}(1,39)=20.088, p<.001, \eta_{p}{ }^{2}=.340\right.$. Fig.5, panel C). JTs were significantly faster after participants observed a fully congruent virtual action $(\mathrm{M}+\mathrm{G}+$ : 
$0.829 \pm 0.015)$ as compared to when they observed any of the possible types of erroneous feedback (all $p s<.008$; all $d s>.272$ ). Moreover, JTs were significantly slower when participants observed a congruent movement with a missed goal (M+G-: $0.884 \pm 0.017)$ as compared to the other two types of erroneous feedback, M-G- $(0.857 \pm 0.017, p=.027, d=0.244)$ and $\mathrm{M}-\mathrm{G}+(0.860 \pm 0.018, d=$ $0.215)$ respectively, even though the latter difference was only marginally significant $(p=.055)$. JTs following M-G+ and M-G- observation did not differ $(p=.991, d=0.023)$. The Anova on JTs did not show any other significant main or interaction effects (all $F s<3.885$, all $p s>.056)$.

\section{Please insert Fig. 5 about here}

\section{4 post observation Reaction Times (poRTs)}

The Anova on poRTs revealed three main effects. Firstly, we found a main effect of factor Movement $\left(\mathrm{F}(1,39)=5.317, p=.027, \eta_{p}{ }^{2}=.120\right.$. Fig. 6, panel A) with slower RTs following the observation of an incongruent (M-: $0.772 \pm 0.018)$ as compared to a congruent movement $(\mathrm{M}+: 0.768 \pm 0.018)$. Secondly, the Anova revealed a main effect of factor Goal $\left(\mathrm{F}(1,39)=5.092, p=.030, \eta_{p}{ }^{2}=0.115\right.$. Fig. 6, panel B) with slower RTs in the trial immediately following a missed (G-: $0.773 \pm 0.018)$ as compared to an achieved goal $(\mathrm{G}+: 0.767 \pm 0.017)$. Finally, the Anova also revealed a main effect of factor Context $\left(\mathrm{F}(1,39)=4.590, p=.038, \eta_{p}{ }^{2}=.105\right)$ : RTs were significantly slower after observing any type of virtual feedback in the free (Free: $0.784 \pm 0.022$ ) as compared to the cued block (Cued: $0.756 \pm 0.016$ ). The Anova on poRTs did not reveal any other main or interaction effects (all $F_{S}<$ 2.121, all $p s>.153)$.

The effect of factor Context suggested that participants were generally faster in performing a button press in the cued as compared to the free block. To check for this, we compared the reaction times in the two blocks, without sorting them according to the type of feedback in the previous trial. We performed this comparison by means of a paired samples t-test on the mean reaction times for each subject in the two blocks (square root transformation was applied, consistently with other analyses on RTs). The t-test was significant $(\mathrm{t}(39)=2.218, p=.032, d=.247)$ indicating that participants performed faster actions in the cued block (Cued: $0.756 \pm 0.016)$ than in the free block (Free: 0.786 $\pm 0.022)$.

To make sure that the difference between the reaction times in the two contexts could not explain the pattern of results revealed by the analysis of Synchrony Judgments we performed a correlation analysis between Synchrony Judgments and Reaction Times. We hypothesized that, given that in the 
Free context participants reported more synchrony and performed actions more slowly, participants with slower reaction times should also be those reporting the higher synchrony and vice versa. Hence, for each subject we calculated the mean reported Synchrony and the mean reaction time by averaging all the conditions and performed a linear correlation of the two measures. Synchrony Judgments and Reaction Times were not correlated $(r=.117 ; \mathrm{p}=.47)$, suggesting that the effects of our experimental factors on synchrony judgments were not associated with reaction times.

\section{Please insert Fig. 6 about here}

\subsection{Control question analysis}

To check that participants were aware of the disposition of the colors when they performed a button press, we conducted an analysis of the control question collected during the SJ session. We calculated the accuracy for each participant in both free and cued blocks. Participants' responded correctly to the control question on average $77.3 \%$ ( \pm S.E.M: $3.1 \%)$ of the times in the free block and $71.1 \%( \pm$ S.E.M: $2.7 \%$ ) of the times in the cued block. We then compared accuracy scores for both free and cued blocks against chance (50\%) by means of two separate one-sample t-tests. Participants were significantly better than chance both in free $(\mathrm{t}(39)=8.891, p<.001, d=1.406)$ and in the cued blocks $(\mathrm{t}(39)=7.917, p<.001, d=1.252)$, which suggests that they were aware of the color disposition when they performed a button press in both free and cued contexts of action.

\section{DISCUSSION}

The aim of this study was to investigate the effects of movement and goal-related prediction errors on implicit and explicit components of SoA within free and cued contexts of action, and if they both lead to behavioral adjustments. To do this, we modified our recently developed paradigm (i.e., SoAGAME, Villa et al., 2018) so that both free and cued actions were possible. Participants performed simple goal-directed actions while they observed similar or different virtual actions represented on a screen from a first-person perspective. We collected both indirect (i.e., SJs) and direct (i.e., JoC) measures of SoA. We also measured behavioral adjustments due to the observation of virtual actions by calculating the amount of time participants took to provide synchrony judgments - i.e., JTs - and to perform a new action after observation of each type of virtual action - i.e., poRTs.

\subsection{Freedom to act enhances implicit, but not explicit SoA}


As expected, the analysis of SJs revealed that participants tended to perceive a virtual action as more synchronous to their own actions when they freely decided which action to perform as compared to when they followed an external cue (main effect of Context). Specifically, this effect was observed only when a delay of 150 or $300 \mathrm{~ms}$ was introduced between real and virtual action and not when the virtual action was simultaneous to the button press (Context $\mathrm{x}$ Delay interaction). Interestingly, we did not observe a similar effect in the analysis of JoC, suggesting that implicit, but not explicit SoA is enhanced by freedom to act. Our findings are in line with recent studies that reported stronger binding between action and outcome - and hence stronger implicit SoA - in a context of freedom of choice, as compared to actions performed following external instructions (Barlas et al., 2017, 2018; Barlas \& Kopp, 2018; Barlas \& Obhi, 2013; Caspar, Christensen, et al., 2016; Caspar et al., 2018, 2017). In particular, the interaction we found between factors Context and Delay is strikingly similar to the one reported by Barlas and colleagues (2017). In their case, stronger binding was observed for free as compared to cued actions, but this effect was observable only when the delays between action and outcome were longer. Our data suggest that information about the context of actions (free choice vs environmental demands) may contribute to SoA when evidence in favor of oneself as the cause of actions is reduced by other factors, such as low temporal contiguity between one's action and the external consequences. A similar interpretation was provided before to explain the contribution of active control over movements for the Sense of Ownership (SoO) - i.e., the sense that my body is 'my own': information about the executed movements may become relevant only when SoO is reduced by the observation of a morphologically incongruent limb (Brugada-Ramentol, Clemens, \& de Polavieja, 2019. But see also Burin et al., 2017, 2015 on the role of movements for the SoO). However, differently from previous studies (Barlas et al., 2017, 2018; Barlas \& Kopp, 2018; Wenke et al., 2010), our data do not support the finding that freedom to act enhances SoA also at an explicit level. Some methodological differences may account for this. For instance, in previous studies participants performed actions finalized at producing outcomes in the external environment, such as eliciting a tone or the appearance of an object on screen. Here, participants observed a virtual action from a first-person perspective. Hence, it is possible that the manipulations of movement and goal present in our task may have been more relevant cues to explicit SoA than freedom to act (see section 4.2).

It is unlikely that the effects of freedom to act on implicit SoA may be due to the fact that participants performed the actions more slowly in the free than in the cued context as revealed by the analysis of reaction times. As a matter of fact, the significant interaction between Context and Delay found in the analysis of Synchrony Judgments is not compatible with this interpretation. Indeed, if the difference between the two contexts was due to a difference in reaction times, it is not clear why this 
would take place only for longer delays between the executed and virtual action, and not also for virtual actions that were simultaneous to the button press. In addition, we did not find any significant correlation between Synchrony Judgments and Reaction Times as shown by the analysis reported in section 3.4 .

One could also argue that the effects of freedom to act on Synchrony Judgments could be due to the fact that participants may have paid more attention to the events taking place in the virtual scenario when freely choosing which color to press. However, the analysis of the control question (see section 3.5) revealed that participants were better than chance in recognizing changes of the disposition of the colors in both contexts, suggesting that participants were paying attention to the virtual actions both when performing actions freely and following cues. We therefore argue that participants experienced a genuine increase of implicit SoA under a context of freedom to act.

\subsection{Movement-related prediction errors reduce both implicit and explicit SoA, while goal- related prediction errors impair only explicit SoA}

In addition to the effects of freedom to act on the feeling of control, our data show that other actioncues contribute to implicit and explicit SoA. Participants perceived a virtual action as more synchronous to their own when the virtual finger moved in their same direction $(\mathrm{M}+)$, as compared to when the virtual finger moved in the opposite direction (M-, main effect of Movement). In addition, SJs were reduced when a delay was introduced between the executed and observed action (main effect of factor Delay). These results partly overlapped with those obtained analyzing JoC. Participants reported higher control when the virtual finger moved in the same as compared to the opposite direction and importantly also when the virtual hand pressed the virtual button of the expected color $\left(\mathrm{G}^{+}\right)$as compared to the unexpected color (G-). In addition, participants felt less in control when longer delays between real and virtual action were introduced (main effect of Delay). Indeed, movement and the temporal contiguity between action and the resulting effect appear to influence both implicit and explicit SoA. Our data suggest that information about movement may be a pivotal source of SoA modulation. This is consistent with previous studies that found that movement congruency influenced SoA both when indirect (Caspar, Desantis, et al., 2016) and direct measures of SoA were employed (Daprati et al., 1997; Farrer et al., 2008; Fourneret \& Jeannerod, 1998; Padrao et al., 2016; van den Bos \& Jeannerod, 2002). Our results are also in line with previous studies that show a reduction of SoA when introducing a delay between executed and observed actions, and between an action and its outcome (Farrer et al., 2008; Franck et al., 2001; Sato \& Yasuda, 2005; Shanks, Pearson, \& Dickinson, 1989; Weiss et al., 2014). In contrast with the effects of movement 
information, our data also suggest that the influence of goal achievement may be limited to explicit SoA. The fact that the failure to achieve the goal of the action did not reduce implicit SoA may be surprising in light of previous studies that employed intentional binding measures (Barlas \& Kopp, 2018; Caspar, Desantis, et al., 2016) and of our previous study (Villa et al., 2018). There, we reported that the failure to achieve the goal of an action reduced SoA, but only when real and virtual action took place simultaneously or with a very short delay $(+75 \mathrm{~ms})$. Here we did not observe the same pattern of results, but some methodological differences may account for this seeming discrepancy. First, in the present study the goal of the action in the cued block was assigned randomly in each trial, while in our previous study participants were asked to press a button of a cued color (blue/yellow) for a long series of trials (around 250). Additionally, in this study each type of virtual feedback was observed an equal number of times ( $25 \%$ of trials), while in the previous study participants observed a fully correct feedback $(\mathrm{M}+\mathrm{G}+)$ in $50 \%$ of trials, and each type of erroneous feedback $(\mathrm{M}+\mathrm{G}-, \mathrm{M}-$ G+, M-G-) in $16 \%$ of trials. Hence, participants to our previous study may have formed a stronger association between action and outcome that resulted in a higher influence of information about goal achievement on SoA with respect to the current study. This interpretation is consistent with a cueintegration theory of SoA (Moore \& Fletcher, 2012. See below). Moreover, it should also be noted that other studies failed to find any effect of outcome congruency on intentional binding (Desantis, Hughes, \& Waszak, 2012; Haering \& Kiesel, 2014). Whether implicit SoA is modulated by goalrelated prediction errors remains an open question that should be further investigated in future studies. Interestingly, goal-related prediction errors were instead effective in modulating explicit SoA. This result is in line with previous studies that reported a reduction of explicit SoA for unexpected action outcomes (David et al., 2016; Kühn et al., 2011; Sato \& Yasuda, 2005). That goal information modulated explicit but not implicit SoA is compatible with the proposal that inferential processes are involved in the formation of explicit beliefs about control (Synofzik et al., 2008a, 2008b; Wegner \& Wheatley, 1999) and with the fact that individuals tend to view themselves as the cause of successful outcomes, and to attribute failures to external factors (Arkin et al., 1980; Miller \& Ross, 1975). This result is also in line with the findings of a recent study by Pezzetta and colleagues, who reported that when participants passively observed a goal-directed action in a fully immersive virtual scenario - a reaching movement to grasp a glass they experienced more control over the virtual action when the virtual hand successfully grasped the glass as compared to when it failed to do so (Pezzetta et al., 2018). Importantly, the proportion of failures (75\%) was higher than the proportion of successes (25\%), which suggests that individual can experience explicit SoA even when the probability of goal achievement is low.

Overall, the different effects of movement and goal information may be compatible with recent 
models that explain how various sources of information contribute to SoA (Moore \& Fletcher, 2012; Synofzik et al., 2008a, 2008b). Moore and Fletcher (2012) proposed a Bayesian model in which multiple action cues are weighted according to their reliability, i.e. to their effectiveness in identifying oneself or an external source as the cause of an event. Given our experimental design, the probability of observing a movement or goal-related prediction error was $50 \%$. This may have different implications for movement and goal information and for implicit and explicit SoA. With respect to movement information, participants may have had strong prior predictions about the way the virtual movement unfolded once they performed the action, since control of one's own body is generally part of everyday experience. Hence, movement-related prediction errors may have been considered by participants an effective source of information that could modulate both implicit and explicit SoA. On the other hand, feeling in control of events in the external environment may require the formation of a stable association between action and outcome (Moore, Lagnado, Deal, \& Haggard, 2009). Given the high probability of failure in achieving the goal in our task, participants may have considered goal information as ineffective in modulating their implicit SoA, that may mostly rely on non-conceptual sensorimotor processes (Synofzik et al., 2008a). Nevertheless, goal-related prediction errors may have been effective in reducing explicit SoA, which may rely also on conscious thoughts about causality.

Importantly, our results do not provide support to the possibility that movement and goal-related prediction errors may exert a different influence on SoA respectively in free and cued actions. Indeed, we did not find any significant interactions between factors Context, Movement and Goal in SJs or JoC analyses. Although conclusions from null results should be extremely cautious, it is nonetheless interesting to note that a similar pattern of results was also reported by Barlas and colleagues (2018). In their study, freedom to act enhanced SoA, while observation of an unexpected outcome reduced it, but these two effects did not interact. We extend Barlas and colleagues conclusions by showing that movement and goal-related prediction errors do not exert a different influence on SoA in free and cued action. Contrary to our hypothesis, information about achievement of the actions' goal does not appear to influence SoA more in free as compared to cued actions as suggested by previous studies (Beck, Di Costa, \& Haggard, 2017; Borhani, Beck, \& Haggard, 2017). Our results are similar to those obtained by Caspar and colleagues, who demonstrated that binding between action and outcome is reduced by the presence of a context of coercion, and it is enhanced by freedom to act irrespective of whether actions resulted in a more or less severe event for another individual (Caspar, Christensen, et al., 2016; Caspar et al., 2018, 2017). Thus, our results support the notion that freedom to act itself may be linked to an enhancement of (implicit) SoA, irrespective of the consequences in the external environment. 


\subsection{Behavioral adjustments follow both movement and goal-related prediction errors}

In addition to modulation of participants' SoA, we also found evidence that movement and goalrelated prediction errors had an influence on their motor performance. Participants were faster in providing a Synchrony Judgment (i.e., JTs) when they observed a fully correct feedback as compared to all types of erroneous feedback (M+G-, M-G+, M-G-, Movement $\mathrm{x}$ Goal interaction). Interestingly, the feedback associated to slower JTs was the one where participants observed a congruent movement which did not achieve the goal $(\mathrm{M}+\mathrm{G}-)$. This indicates that goalrelated prediction errors affected participants behavior even if the movement was congruent.

Evidence for behavioral adjustments following movement and goal-related prediction errors also comes from the analysis of poRTs. Indeed, participants were slower in performing a new action after observing both a failure to achieve the goal of the action and an incongruent movement in the previous trial. Overall, the analyses of JTs and poRTs suggest that not only SoA, but also participants' behavior was affected by prediction errors. The slowing observed in both measures may be similar to behavioral adjustments that occur after a real error, in particular the Post Error Slowing (Danielmeier \& Ullsperger, 2011; Fusco et al., 2018; Ullsperger et al., 2014). Our findings are also in line with previous evidence suggesting that prediction errors and unexpected action-related visual events (in our case the observation of congruent or incongruent virtual actions from a first-person perspective) lead to behavioral adjustments (Gentsch et al., 2009; Padrao et al., 2016; Wessel \& Aron, 2013; Wessel et al., 2012).

Interestingly, despite movement appeared to be a more relevant cue to SoA as compared to goal achievement (at least for implicit SoA), the latter appeared to exert a strong influence on behavioral adjustments, even stronger than movement information as suggested by the analysis of JTs. This further suggests that the effect of prediction errors on SoA is influenced by other factors, such as the reliability of a specific action cue (Moore \& Fletcher, 2012; Synofzik et al., 2008a, 2008b). Finally, we did not find any evidence of a significant interaction between movement and goal-related prediction errors and free or cued contexts of action. This suggests that erroneous or unexpected consequences of free and cued actions may lead to similar behavioral adjustments.

\section{CONCLUSION}

In this study we investigated the effects of movement and goal-related prediction-errors on implicit and explicit components of the Sense of Agency and on behavioral adjustments when participants performed freely chosen and cued actions. Our data support the notion that freedom to act enhances SoA, but we show that its influence may be limited to implicit SoA and to conditions where the 
temporal contiguity between one's actions and the external consequences is low. Moreover, our data indicate that that information about movement execution may be the pivotal cue to both implicit and explicit SoA, while goal achievement appears to mostly influence explicit SoA. We hypothesize that the contribution of goal information to implicit SoA may increase in case of a more stable association between action and outcome (Moore et al., 2009) or in case the goal was endowed with an affective or rewarding valence (Takahata et al., 2012; Yoshie \& Haggard, 2013). Future studies may tackle these issues. Importantly, our data suggest that the effects on SoA of freedom to act and of movement and goal-related prediction errors are independent. Finally, we show that movement and goal related prediction errors may generate behavioral adjustments.

Conflict of Interest: The authors declare that they have no conflict of interest.

Compliance with ethical standards: The experimental protocol was approved by the ethics committee of Fondazione Santa Lucia (Prot. CE/PROG. 686) and was performed in accordance with the 1964 declaration of Helsinki. All participants read and provided written informed consent prior to taking part in the study.

Data availability statement The datasets generated during and/or analyzed during the current study are available from the corresponding author on reasonable request. 


\section{REFERENCES}

Aarts, H., Bijleveld, E., Custers, R., Dogge, M., Deelder, M., Schutter, D., \& van Haren, N. E. M. (2012). Positive priming and intentional binding: Eye-blink rate predicts reward information effects on the sense of agency. Social Neuroscience, 7(1), 105-112. https://doi.org/10.1080/17470919.2011.590602

Arkin, R. M., Appelman, A. J., \& Burger, J. M. (1980). Social anxiety, self-presentation, and the self-serving bias in causal attribution. Journal of Personality and Social Psychology, 38(1), 23-35. https://doi.org/10.1037/0022-3514.38.1.23

Barlas, Z., Hockley, W. E., \& Obhi, S. S. (2017). The effects of freedom of choice in action selection on perceived mental effort and the sense of agency. Acta Psychologica, 180(August), 122-129. https://doi.org/10.1016/j.actpsy.2017.09.004

Barlas, Z., Hockley, W. E., \& Obhi, S. S. (2018). Effects of free choice and outcome valence on the sense of agency: evidence from measures of intentional binding and feelings of control. Experimental Brain Research, 236(1), 129-139. https://doi.org/10.1007/s00221-017-5112-3

Barlas, Z., \& Kopp, S. (2018). Action Choice and Outcome Congruency Independently Affect Intentional Binding and Feeling of Control Judgments. Frontiers in Human Neuroscience, 12(April), 1-10. https://doi.org/10.3389/fnhum.2018.00137

Barlas, Z., \& Obhi, S. S. (2013). Freedom, choice, and the sense of agency. Frontiers in Human Neuroscience, 7(August), 514. https://doi.org/10.3389/fnhum.2013.00514

Beck, B., Di Costa, S., \& Haggard, P. (2017). Having control over the external world increases the implicit sense of agency. Cognition, 162, 54-60. https://doi.org/10.1016/j.cognition.2017.02.002

Blakemore, S.-J., \& Frith, C. D. (2003). Self-awareness and action. Current Opinion in Neurobiology, 13(2), 219-224. https://doi.org/10.1016/S0959-4388(03)00043-6

Blakemore, S.-J., \& Sirigu, A. (2003). Action prediction in the cerebellum and in the parietal lobe. Experimental Brain Research, 153(2), 239-245. https://doi.org/10.1007/s00221-003-1597-z

Blakemore, S.-J., Wolpert, D. M., \& Frith, C. D. (2002). Abnormalities in the awareness of action. Trends in Cognitive Sciences, 6(6), 237-242. https://doi.org/10.1016/S1364-6613(02)01907-1

Borhani, K., Beck, B., \& Haggard, P. (2017). Choosing, Doing, and Controlling: Implicit Sense of Agency Over Somatosensory Events. Psychological Science, 28(7), 882-893. https://doi.org/10.1177/0956797617697693

Braun, N., Thorne, J. D., Hildebrandt, H., \& Debener, S. (2014). Interplay of Agency and Ownership: The Intentional Binding and Rubber Hand Illusion Paradigm Combined. PLoS ONE, 9(11), e111967. https://doi.org/10.1371/journal.pone.0111967

Brugada-Ramentol, V., Clemens, I., \& de Polavieja, G. G. (2019). Active control as evidence in favor of sense of ownership in the moving Virtual Hand Illusion. Consciousness and Cognition, 71(August 2018), 123-135. https://doi.org/10.1016/j.concog.2019.04.003

Burin, D., Garbarini, F., Bruno, V., Fossataro, C., Destefanis, C., Berti, A., \& Pia, L. (2017). Movements and body ownership: Evidence from the rubber hand illusion after mechanical limb immobilization. Neuropsychologia, 107(June 2018), 41-47. https://doi.org/10.1016/j.neuropsychologia.2017.11.004

Burin, D., Livelli, A., Garbarini, F., Fossataro, C., Folegatti, A., Gindri, P., \& Pia, L. (2015). Are 
movements necessary for the sense of body ownership? evidence from the rubber hand illusion in pure hemiplegic patients. PLOS ONE, 10(3), 1-12.

https://doi.org/10.1371/journal.pone.0117155

Campbell, J. I. D., \& Thompson, V. A. (2012). MorePower 6.0 for ANOVA with relational confidence intervals and Bayesian analysis. Behavior Research Methods, 44(4), 1255-1265. https://doi.org/10.3758/s13428-012-0186-0

Caspar, E. A., Christensen, J. F., Cleeremans, A., \& Haggard, P. (2016). Coercion Changes the Sense of Agency in the Human Brain. Current Biology, 26(5), 585-592. https://doi.org/10.1016/j.cub.2015.12.067

Caspar, E. A., Cleeremans, A., \& Haggard, P. (2015). The relationship between human agency and embodiment. Consciousness and Cognition, 33, 226-236. https://doi.org/10.1016/j.concog.2015.01.007

Caspar, E. A., Cleeremans, A., \& Haggard, P. (2018). Only giving orders? An experimental study of the sense of agency when giving or receiving commands. PLOS ONE, 13(9), e0204027. https://doi.org/10.1371/journal.pone.0204027

Caspar, E. A., Desantis, A., Dienes, Z., Cleeremans, A., \& Haggard, P. (2016). The sense of agency as tracking control. PLoS ONE, 11(10), e0163892. https://doi.org/10.1371/journal.pone.0163892

Caspar, E. A., Vuillaume, L., Magalhães De Saldanha da Gama, P. A., \& Cleeremans, A. (2017). The Influence of (Dis)belief in Free Will on Immoral Behavior. Frontiers in Psychology, 8(JAN), 1-9. https://doi.org/10.3389/fpsyg.2017.00020

Danielmeier, C., \& Ullsperger, M. (2011). Post-Error Adjustments. Frontiers in Psychology, 2(SEP). https://doi.org/10.3389/fpsyg.2011.00233

Daprati, E., Franck, N., Georgieff, N., Proust, J., Pacherie, E., Dalery, J., \& Jeannerod, M. (1997). Looking for the agent: an investigation into consciousness of action and self-consciousness in schizophrenic patients. Cognition, 65(1), 71-86. https://doi.org/10.1016/S00100277(97)00039-5

David, N., Skoruppa, S., Gulberti, A., Schultz, J., \& Engel, A. K. (2016). The Sense of Agency Is More Sensitive to Manipulations of Outcome than Movement-Related Feedback Irrespective of Sensory Modality. PLOS ONE, 11(8), e0161156. https://doi.org/10.1371/journal.pone.0161156

den Ouden, H. E. M., Kok, P., \& de Lange, F. P. (2012). How Prediction Errors Shape Perception, Attention, and Motivation. Frontiers in Psychology, 3(DEC), 1-12. https://doi.org/10.3389/fpsyg.2012.00548

Desantis, A., Hughes, G., \& Waszak, F. (2012). Intentional Binding Is Driven by the Mere Presence of an Action and Not by Motor Prediction. PLoS ONE, 7(1), e29557. https://doi.org/10.1371/journal.pone.0029557

Farrer, C., Frey, S. H., Van Horn, J. D., Tunik, E., Turk, D., Inati, S., \& Grafton, S. T. (2008). The Angular Gyrus Computes Action Awareness Representations. Cerebral Cortex, 18(2), 254261. https://doi.org/10.1093/cercor/bhm050

Fischer, R. (2004). Standardization to Account for Cross-Cultural Response Bias. Journal of CrossCultural Psychology, 35(3), 263-282. https://doi.org/10.1177/0022022104264122

Fischer, R., \& Milfont, T. L. (2010). Standardization in psychological research. International 
Journal of Psychological Research, 3(1 SE-Research Article). https://doi.org/10.21500/20112084.852

Fourneret, P., \& Jeannerod, M. (1998). Limited conscious monitoring of motor performance in normal subjects. Neuropsychologia, 36(11), 1133-1140. https://doi.org/10.1016/S00283932(98)00006-2

Franck, N., Farrer, C., Georgieff, N., Marie-Cardine, M., Daléry, J., D’Amato, T., \& Jeannerod, M. (2001). Defective Recognition of One's Own Actions in Patients With Schizophrenia. American Journal of Psychiatry, 158(3), 454-459. https://doi.org/10.1176/appi.ajp.158.3.454

Fried, I., Haggard, P., He, B. J., \& Schurger, A. (2017). Volition and Action in the Human Brain: Processes, Pathologies, and Reasons. The Journal of Neuroscience, 37(45), 10842-10847. https://doi.org/10.1523/JNEUROSCI.2584-17.2017

Friston, K. (2010). The free-energy principle: A unified brain theory? Nature Reviews Neuroscience, 11(2), 127-138. https://doi.org/10.1038/nrn2787

Friston, K. (2011). What Is Optimal about Motor Control? Neuron, 72(3), 488-498. https://doi.org/10.1016/j.neuron.2011.10.018

Friston, K. (2012). Prediction , perception and agency. International Journal of Psychophysiology, 83(2), 248-252. https://doi.org/10.1016/j.ijpsycho.2011.11.014

Friston, K., Samothrakis, S., \& Montague, R. (2012). Active inference and agency: optimal control without cost functions. Biological Cybernetics, 106(8-9), 523-541. https://doi.org/10.1007/s00422-012-0512-8

Frith, C. D. (2013). The psychology of volition. Experimental Brain Research, 229(3), 289-299. https://doi.org/10.1007/s00221-013-3407-6

Frith, C. D., Blakemore, S.-J., \& Wolpert, D. M. (2000). Abnormalities in the awareness and control of action. Philosophical Transactions of the Royal Society B: Biological Sciences, 355(1404), 1771-1788. https://doi.org/10.1098/rstb.2000.0734

Fusco, G., Scandola, M., Feurra, M., Pavone, E. F., Rossi, S., \& Aglioti, S. M. (2018). Midfrontal theta transcranial alternating current stimulation modulates behavioural adjustment after error execution. European Journal of Neuroscience, 48(10), 3159-3170. https://doi.org/10.1111/ejn.14174

Gentsch, A., Ullsperger, P., \& Ullsperger, M. (2009). Dissociable medial frontal negativities from a common monitoring system for self- and externally caused failure of goal achievement. NeuroImage, 47(4), 2023-2030. https://doi.org/10.1016/j.neuroimage.2009.05.064

Haering, C., \& Kiesel, A. (2014). Intentional Binding is independent of the validity of the action effect's identity. Acta Psychologica, 152, 109-119. https://doi.org/10.1016/j.actpsy.2014.07.015

Haggard, P. (2017). Sense of agency in the human brain. Nature Reviews Neuroscience, 18(4), 196207. https://doi.org/10.1038/nrn.2017.14

Haggard, P., Clark, S., \& Kalogeras, J. (2002). Voluntary action and conscious awareness. Nature Neuroscience, 5(4), 382-385. https://doi.org/10.1038/nn827

Kalckert, A., \& Ehrsson, H. H. (2012). Moving a Rubber Hand that Feels Like Your Own: A Dissociation of Ownership and Agency. Frontiers in Human Neuroscience, 6(March), 1-14. https://doi.org/10.3389/fnhum.2012.00040 
Kalckert, A., \& Ehrsson, H. H. (2014). The moving rubber hand illusion revisited: Comparing movements and visuotactile stimulation to induce illusory ownership. Consciousness and Cognition, 26(1), 117-132. https://doi.org/10.1016/j.concog.2014.02.003

Kirsch, W., Kunde, W., \& Herbort, O. (2019). Intentional binding is unrelated to action intention. Journal of Experimental Psychology: Human Perception and Performance, 45(3), 378-385. https://doi.org/10.1037/xhp0000612

Kühn, S., Nenchev, I., Haggard, P., Brass, M., Gallinat, J., \& Voss, M. (2011). Whodunnit? Electrophysiological Correlates of Agency Judgements. PLoS ONE, 6(12), e28657. https://doi.org/10.1371/journal.pone.0028657

Miller, D. T., \& Ross, M. (1975). Self-serving biases in the attribution of causality: Fact or fiction? Psychological Bulletin, 82(2), 213-225. https://doi.org/10.1037/h0076486

Monti, A., Porciello, G., Tieri, G., \& Aglioti, S. M. (2020). The "embreathment" illusion highlights the role of breathing in corporeal awareness. Journal of Neurophysiology, 123(1), 420-427. https://doi.org/10.1152/jn.00617.2019

Moore, J. W., \& Fletcher, P. C. (2012). Sense of agency in health and disease: A review of cue integration approaches. Consciousness and Cognition, 21(1), 59-68. https://doi.org/10.1016/j.concog.2011.08.010

Moore, J. W., Lagnado, D., Deal, D. C., \& Haggard, P. (2009). Feelings of control: Contingency determines experience of action. Cognition, 110(2), 279-283. https://doi.org/10.1016/j.cognition.2008.11.006

Moore, J. W., \& Obhi, S. S. (2012). Intentional binding and the sense of agency: A review. Consciousness and Cognition, 21(1), 546-561. https://doi.org/10.1016/j.concog.2011.12.002

Moreau, Q., Candidi, M., Era, V., Tieri, G., \& Aglioti, S. M. (2019). Frontal and occipito-temporal Theta activity as marker of error monitoring in Human-Avatar joint performance. BioRxiv, 402149. https://doi.org/10.1101/402149

Osumi, M., Nobusako, S., Zama, T., Yokotani, N., Shimada, S., Maeda, T., \& Morioka, S. (2019). The relationship and difference between delay detection ability and judgment of sense of agency. PLOS ONE, 14(7), e0219222. https://doi.org/10.1371/journal.pone.0219222

Padrao, G., Gonzalez-Franco, M., Sanchez-Vives, M. V., Slater, M., \& Rodriguez-Fornells, A. (2016). Violating body movement semantics: Neural signatures of self-generated and externalgenerated errors. NeuroImage, 124, 147-156. https://doi.org/10.1016/j.neuroimage.2015.08.022

Pavone, E. F., Tieri, G., Rizza, G., Tidoni, E., Grisoni, L., \& Aglioti, S. M. (2016). Embodying Others in Immersive Virtual Reality: Electro-Cortical Signatures of Monitoring the Errors in the Actions of an Avatar Seen from a First-Person Perspective. Journal of Neuroscience, 36(2), 268-279. https://doi.org/10.1523/JNEUROSCI.0494-15.2016

Pezzetta, R., Nicolardi, V., Tidoni, E., \& Aglioti, S. M. (2018). Error, rather than its probability, elicits specific electrocortical signatures: a combined EEG-immersive virtual reality study of action observation. Journal of Neurophysiology, 120(3), 1107-1118. https://doi.org/10.1152/jn.00130.2018

Poonian, S. K., \& Cunnington, R. (2013). Intentional binding in self-made and observed actions. Experimental Brain Research, 229(3), 419-427. https://doi.org/10.1007/s00221-013-3505-5

Salomon, R., Fernandez, N. B., Van Elk, M., Vachicouras, N., Sabatier, F., Tychinskaya, A., ... 
Blanke, O. (2016). Changing motor perception by sensorimotor conflicts and body ownership. Scientific Reports, 6(December 2015), 1-13. https://doi.org/10.1038/srep25847

Sato, A., \& Yasuda, A. (2005). Illusion of sense of self-agency: discrepancy between the predicted and actual sensory consequences of actions modulates the sense of self-agency, but not the sense of self-ownership. Cognition, 94(3), 241-255. https://doi.org/10.1016/j.cognition.2004.04.003

Shanks, D. R., Pearson, S. M., \& Dickinson, A. (1989). Temporal Contiguity and the Judgement of Causality by Human Subjects. The Quarterly Journal of Experimental Psychology Section B, 41(2), 139-159. https://doi.org/10.1080/14640748908401189

Suzuki, K., Lush, P., Seth, A. K., \& Roseboom, W. (2019). Intentional Binding Without Intentional Action. Psychological Science, 30(6), 842-853. https://doi.org/10.1177/0956797619842191

Synofzik, M., Vosgerau, G., \& Newen, A. (2008a). Beyond the comparator model: A multifactorial two-step account of agency. Consciousness and Cognition, 17(1), 219-239. https://doi.org/10.1016/j.concog.2007.03.010

Synofzik, M., Vosgerau, G., \& Newen, A. (2008b). I move, therefore I am: A new theoretical framework to investigate agency and ownership. Consciousness and Cognition, 17(2), 411424. https://doi.org/10.1016/j.concog.2008.03.008

Synofzik, M., Vosgerau, G., \& Voss, M. (2013). The experience of agency: an interplay between prediction and postdiction. Frontiers in Psychology, 4(MAR), 1-8. https://doi.org/10.3389/fpsyg.2013.00127

Takahata, K., Takahashi, H., Maeda, T., Umeda, S., Suhara, T., Mimura, M., \& Kato, M. (2012). It's Not My Fault: Postdictive Modulation of Intentional Binding by Monetary Gains and Losses. PLoS ONE, 7(12), 1-8. https://doi.org/10.1371/journal.pone.0053421

Tieri, G., Tidoni, E., Pavone, E. F., \& Aglioti, S. M. (2015). Mere observation of body discontinuity affects perceived ownership and vicarious agency over a virtual hand. Experimental Brain Research, 233(4), 1247-1259. https://doi.org/10.1007/s00221-015-4202-3

Tsakiris, M., Longo, M. R., \& Haggard, P. (2010). Having a body versus moving your body: Neural signatures of agency and body-ownership. Neuropsychologia, 48(9), 2740-2749. https://doi.org/10.1016/j.neuropsychologia.2010.05.021

Ullsperger, M., Danielmeier, C., \& Jocham, G. (2014). Neurophysiology of Performance Monitoring and Adaptive Behavior. Physiological Reviews, 94(1), 35-79. https://doi.org/10.1152/physrev.00041.2012

van den Bos, E., \& Jeannerod, M. (2002). Sense of body and sense of action both contribute to selfrecognition. Cognition, 85(2), 177-187. https://doi.org/10.1016/S0010-0277(02)00100-2

Villa, R., Tidoni, E., Porciello, G., \& Aglioti, S. M. (2018). Violation of expectations about movement and goal achievement leads to Sense of Agency reduction. Experimental Brain Research, 236(7), 2123-2135. https://doi.org/10.1007/s00221-018-5286-3

Wegner, D. M., \& Sparrow, B. (2004). Authorship Processing. In M.S. Gazzaniga (Ed.), The New Cognitive Neurosciences (3rd ed., pp. 1201-1209). Cambridge, MA: MIT Press.

Wegner, D. M., Sparrow, B., \& Winerman, L. (2004). Vicarious agency: experiencing control over the movements of others. Journal of Personality and Social Psychology, 86(6), 838-848. https://doi.org/10.1037/0022-3514.86.6.838 
Wegner, D. M., \& Wheatley, T. (1999). Apparent mental causation: Sources of the experience of will. American Psychologist, 54(7), 480-492. https://doi.org/10.1037/0003-066X.54.7.480

Weiss, C., Tsakiris, M., Haggard, P., \& Schütz-Bosbach, S. (2014). Agency in the sensorimotor system and its relation to explicit action awareness. Neuropsychologia, 52(1), 82-92. https://doi.org/10.1016/j.neuropsychologia.2013.09.034

Wenke, D., Fleming, S. M., \& Haggard, P. (2010). Subliminal priming of actions influences sense of control over effects of action. Cognition, 115(1), 26-38. https://doi.org/10.1016/j.cognition.2009.10.016

Wessel, J. R., \& Aron, A. R. (2013). Unexpected Events Induce Motor Slowing via a Brain Mechanism for Action-Stopping with Global Suppressive Effects. Journal of Neuroscience, 33(47), 18481-18491. https://doi.org/10.1523/JNEUROSCI.3456-13.2013

Wessel, J. R., Danielmeier, C., Morton, J. B., \& Ullsperger, M. (2012). Surprise and Error: Common Neuronal Architecture for the Processing of Errors and Novelty. Journal of Neuroscience, 32(22), 7528-7537. https://doi.org/10.1523/JNEUROSCI.6352-11.2012

Wolpe, N., \& Rowe, J. B. (2014a). Beyond the "urge to move": objective measures for the study of agency in the post-Libet era. Frontiers in Human Neuroscience, 8(JUNE), 1-13. https://doi.org/10.3389/fnhum.2014.00450

Wolpe, N., \& Rowe, J. B. (2014b). Beyond the "urge to move": objective measures for the study of agency in the post-Libet era. Frontiers in Human Neuroscience, 8(JUNE), 1-13. https://doi.org/10.3389/fnhum.2014.00450

Wolpert, D., Ghahramani, Z., \& Jordan, M. (1995). An internal model for sensorimotor integration. Science, 269(5232), 1880-1882. https://doi.org/10.1126/science.7569931

Yoshie, M., \& Haggard, P. (2013). Negative Emotional Outcomes Attenuate Sense of Agency over Voluntary Actions. Current Biology, 23(20), 2028-2032. https://doi.org/10.1016/j.cub.2013.08.034 


\section{CAPTIONS TO FIGURES AND TABLES}

Fig. 1 Experimental set-up. Participants performed simple goal-directed actions - to select one of the two virtual buttons according to its color (blue or yellow) and to press the real button in the corresponding position as fast as possible - and observed a virtual hand performing the same or a different action from a first-person perspective (panel a). A screen was placed on a wooden structure in an inclined position, and a hole at the front of the structure allowed participants to place their hand under the screen and hidden from sight. Participants inserted their finger between the two plastic buttons of a custom-made response box (panel b), which allowed to collect downward and upwards movements. In separate blocks, participants could either freely select which color to press (free actions) or follow an imperative cue (cued actions, in the example we show a participant that has to press blue). The virtual action could be simultaneous $(+0 \mathrm{~ms})$ or delayed $(+150,+300 \mathrm{~ms})$ with respect to the real button press. We collected Synchrony Judgments and Judgments of Causation expressed by means of a Visual Analogue Scale as indirect and direct measure of SoA, respectively

Fig. 2 Experimental procedure and virtual stimuli. The experiment was divided in two sessions (panel A): in the SJ session participants were asked to provide Synchrony Judgments between the virtual action and the observed the virtual action. In the JoC session, participants provided Judgments of Causation of the virtual action by means of a Visual Analogue Scale. Participants performed the two sessions in a fixed order - first the SJ session, then the JoC session. Each session was divided in two blocks, whose order was counterbalanced across participants: in the "Free" block participants performed freely chosen actions, in the "Cued" block they followed cues. The structure of each trial was identical for free and cued actions (panel B), with the only difference being the type of symbol that appeared at the beginning of the trial. In the cued block, a yellow or blue circle instructed participants about which color they had to press. In the free block, the circle was half blue and half yellow and reminded participants to choose which color to press. The circle remained visible for 1000 $\mathrm{ms}$ and then disappeared. After a random time comprised between 1000 and $1500 \mathrm{~ms}$ the two virtual buttons flashed for $120 \mathrm{~ms}$, one of them turning blue and the other yellow. Participants pressed the real button in the same position as the selected color with an upward or downward movement and observed a virtual action - i.e., visual feedback - which took place simultaneously $(+0 \mathrm{~ms})$ or delayed $(+150,+300 \mathrm{~ms})$ with respect to the button press. The visual feedback remained visible for $500 \mathrm{~ms}$. After that participants were asked to respond to the agency question (Synchrony Judgments or Judgments of Causation). The inter-trial interval (ITI) was $1000 \mathrm{~ms}$. The possible types of visual feedback are reported in panel C. For simplicity we represent only the case where blue is up and 
yellow is down and the participants presses the blue button. At the center of the panel the four possible types of feedback (M+G+, M+G-, M-G+, M-G-) are represented. To the right of the panel we report the prohibition signal that participants observed in the cued block if they pressed the wrong button (real error)

Fig. 3 Effects of movement, context and delay manipulations on Synchrony Judgments (SJs). The analysis of Synchrony Judgments revealed that participants perceived the visual feedback as more synchronous when the virtual movement was congruent with the real one (main effect of factor Movement; panel A) and when they performed free as compared to cued actions (main effect of factor Context, panel B). Moreover, participants could discriminate delays of increasing duration (main effect of factor delay, panel C). Interestingly, participants perceived free actions as more synchronous with respect to cued ones when delays of $150 \mathrm{~ms}$ or $300 \mathrm{~ms}$ were introduced between real and virtual actions, but not when virtual actions took place simultaneously $(+0 \mathrm{~ms})$ (Action Type X Delay interaction, panel D). Error bars represent the Standard Error of the Mean in all panels

\section{Fig. 4 Effects of Movement, Goal and Delay manipulations on the Judgments of Causation} (JoC). The analysis of JoC revealed three main effects. Participants experienced lower SoA i) when they observed that the virtual index finger moved in the opposite direction as compared to when it moved in their same direction (main effect of factor Movement, panel A); ii) when the color pressed by the virtual hand was not the selected one as compared to when it was the selected one (main effect of factor Goal, Panel B); and iii) for longer delays between real and virtual actions (main effect of factor Delay, panel C)

Fig. 5 Effects of movement, goal and delay manipulations on Judgment Times (JTs). The analysis of JTs showed that participants were significantly faster in providing a Synchrony Judgment when a delay of $300 \mathrm{~ms}$ was introduced between real and virtual action, as compared to when the delay was of $150 \mathrm{~ms}$ or when the virtual action was simultaneous to the real one $(+0 \mathrm{~ms}$. Main effect of factor Delay, panel A). Moreover, JTs were significantly higher when participants observed that the goal of the action was missed as compared to when it was achieved (main effect of factor Goal, panel B). This effect was further explained by a significant interaction between factors Movement and Goal: participants showed faster JTs when they observed a fully congruent virtual action (the virtual index finger moved in the same direction of the participant and pressed the selected color) as compared to all the types of erroneous feedback. Additionally, participants showed slower JTs when they observed a congruent movement and the goal of the action was missed (M+G-) as compared to the other types of erroneous feedback (M-G+, M-G-). Please note that the difference between $\mathrm{M}+\mathrm{G}-$ 
and $\mathrm{M}-\mathrm{G}+$ approximated significance $(p=.055)$. Error bars represent the Standard Error of the Mean in all panels

\section{Fig. 6 Effects of Movement and Goal manipulations on post observation Reaction Times} (poRTs). The analysis of poRTs revealed that participants were significantly slower in performing a button press after observing an incongruent as compared to a congruent movement (main effect of factor Movement, panel A). Moreover, participants were significantly slower in performing a new action after observing that the virtual hand pressed the virtual button of the color they did not select as compared to when the virtual hand pressed the selected color (main effect of factor Goal, panel B). Error bars represent the Standard Error of the Mean in all panels

Table 1 The table reports the mean \pm Standard Error of the Mean of ipsatized Synchrony Judgments for all levels of the interaction between factors Context (Free/Cued) and Delay $(+0 \mathrm{~ms},+150 \mathrm{~ms},+$ $300 \mathrm{~ms})$ 


\section{CAPTIONS TO FIGURES AND TABLES}

Fig. 1 Experimental set-up. Participants performed simple goal-directed actions - to select one of the two virtual buttons according to its color (blue or yellow) and to press the real button in the corresponding position as fast as possible - and observed a virtual hand performing the same or a different action from a first-person perspective (panel a). A screen was placed on a wooden structure in an inclined position, and a hole at the front of the structure allowed participants to place their hand under the screen and hidden from sight. Participants inserted their finger between the two plastic buttons of a custom-made response box (panel b), which allowed to collect downward and upwards movements. In separate blocks, participants could either freely select which color to press (free actions) or follow an imperative cue (cued actions, in the example we show a participant that has to press blue). The virtual action could be simultaneous $(+0 \mathrm{~ms})$ or delayed $(+150,+300 \mathrm{~ms})$ with respect to the real button press. We collected Synchrony Judgments and Judgments of Causation expressed by means of a Visual Analogue Scale as indirect and direct measure of SoA, respectively

Fig. 2 Experimental procedure and virtual stimuli. The experiment was divided in two sessions (panel A): in the SJ session participants were asked to provide Synchrony Judgments between the virtual action and the observed the virtual action. In the JoC session, participants provided Judgments of Causation of the virtual action by means of a Visual Analogue Scale. Participants performed the two sessions in a fixed order - first the SJ session, then the JoC session. Each session was divided in two blocks, whose order was counterbalanced across participants: in the "Free" block participants performed freely chosen actions, in the "Cued" block they followed cues. The structure of each trial was identical for free and cued actions (panel B), with the only difference being the type of symbol that appeared at the beginning of the trial. In the cued block, a yellow or blue circle instructed participants about which color they had to press. In the free block, the circle was half blue and half yellow and reminded participants to choose which color to press. The circle remained visible for 1000 $\mathrm{ms}$ and then disappeared. After a random time comprised between 1000 and $1500 \mathrm{~ms}$ the two virtual buttons flashed for $120 \mathrm{~ms}$, one of them turning blue and the other yellow. Participants pressed the real button in the same position as the selected color with an upward or downward movement and observed a virtual action - i.e., visual feedback - which took place simultaneously $(+0 \mathrm{~ms})$ or delayed $(+150,+300 \mathrm{~ms})$ with respect to the button press. The visual feedback remained visible for $500 \mathrm{~ms}$. After that participants were asked to respond to the agency question (Synchrony Judgments or Judgments of Causation). The inter-trial interval (ITI) was $1000 \mathrm{~ms}$. The possible types of visual feedback are reported in panel C. For simplicity we represent only the case where blue is up and 
yellow is down and the participants presses the blue button. At the center of the panel the four possible types of feedback (M+G+, M+G-, M-G+, M-G-) are represented. To the right of the panel we report the prohibition signal that participants observed in the cued block if they pressed the wrong button (real error)

Fig. 3 Effects of movement, context and delay manipulations on Synchrony Judgments (SJs). The analysis of Synchrony Judgments revealed that participants perceived the visual feedback as more synchronous when the virtual movement was congruent with the real one (main effect of factor Movement; panel A) and when they performed free as compared to cued actions (main effect of factor Context, panel B). Moreover, participants could discriminate delays of increasing duration (main effect of factor delay, panel C). Interestingly, participants perceived free actions as more synchronous with respect to cued ones when delays of $150 \mathrm{~ms}$ or $300 \mathrm{~ms}$ were introduced between real and virtual actions, but not when virtual actions took place simultaneously $(+0 \mathrm{~ms})$ (Action Type X Delay interaction, panel D). Error bars represent the Standard Error of the Mean in all panels

\section{Fig. 4 Effects of Movement, Goal and Delay manipulations on the Judgments of Causation} (JoC). The analysis of JoC revealed three main effects. Participants experienced lower SoA i) when they observed that the virtual index finger moved in the opposite direction as compared to when it moved in their same direction (main effect of factor Movement, panel A); ii) when the color pressed by the virtual hand was not the selected one as compared to when it was the selected one (main effect of factor Goal, Panel B); and iii) for longer delays between real and virtual actions (main effect of factor Delay, panel C)

Fig. 5 Effects of movement, goal and delay manipulations on Judgment Times (JTs). The analysis of JTs showed that participants were significantly faster in providing a Synchrony Judgment when a delay of $300 \mathrm{~ms}$ was introduced between real and virtual action, as compared to when the delay was of $150 \mathrm{~ms}$ or when the virtual action was simultaneous to the real one $(+0 \mathrm{~ms}$. Main effect of factor Delay, panel A). Moreover, JTs were significantly higher when participants observed that the goal of the action was missed as compared to when it was achieved (main effect of factor Goal, panel B). This effect was further explained by a significant interaction between factors Movement and Goal: participants showed faster JTs when they observed a fully congruent virtual action (the virtual index finger moved in the same direction of the participant and pressed the selected color) as compared to all the types of erroneous feedback. Additionally, participants showed slower JTs when they observed a congruent movement and the goal of the action was missed (M+G-) as compared to the other types of erroneous feedback (M-G+, M-G-). Please note that the difference between $\mathrm{M}+\mathrm{G}-$ 
and $\mathrm{M}-\mathrm{G}+$ approximated significance $(p=.055)$. Error bars represent the Standard Error of the Mean in all panels

\section{Fig. 6 Effects of Movement and Goal manipulations on post observation Reaction Times} (poRTs). The analysis of poRTs revealed that participants were significantly slower in performing a button press after observing an incongruent as compared to a congruent movement (main effect of factor Movement, panel A). Moreover, participants were significantly slower in performing a new action after observing that the virtual hand pressed the virtual button of the color they did not select as compared to when the virtual hand pressed the selected color (main effect of factor Goal, panel B). Error bars represent the Standard Error of the Mean in all panels

Table 1 The table reports the mean \pm Standard Error of the Mean of ipsatized Synchrony Judgments for all levels of the interaction between factors Context (Free/Cued) and Delay $(+0 \mathrm{~ms},+150 \mathrm{~ms},+$ $300 \mathrm{~ms})$ 


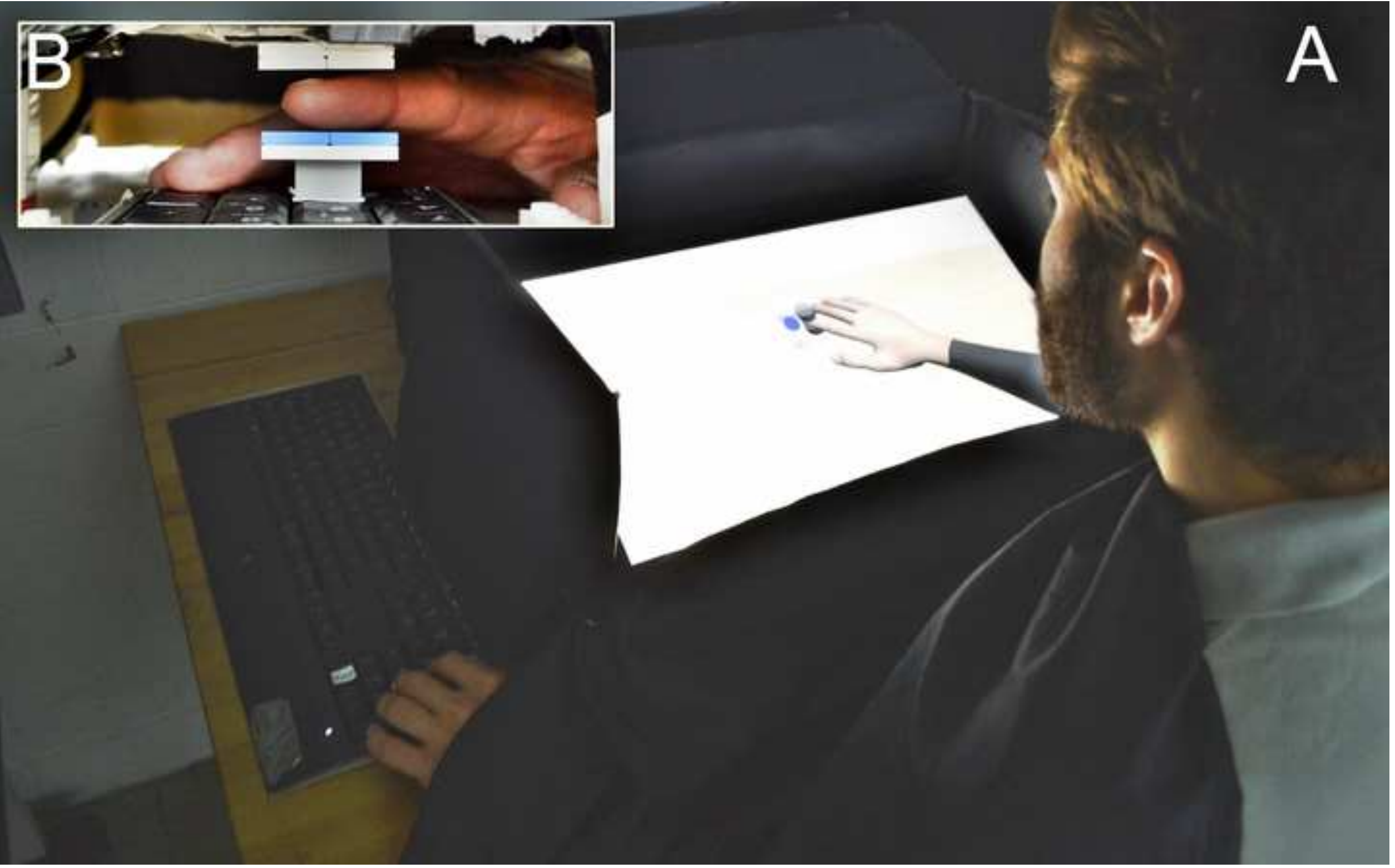


A

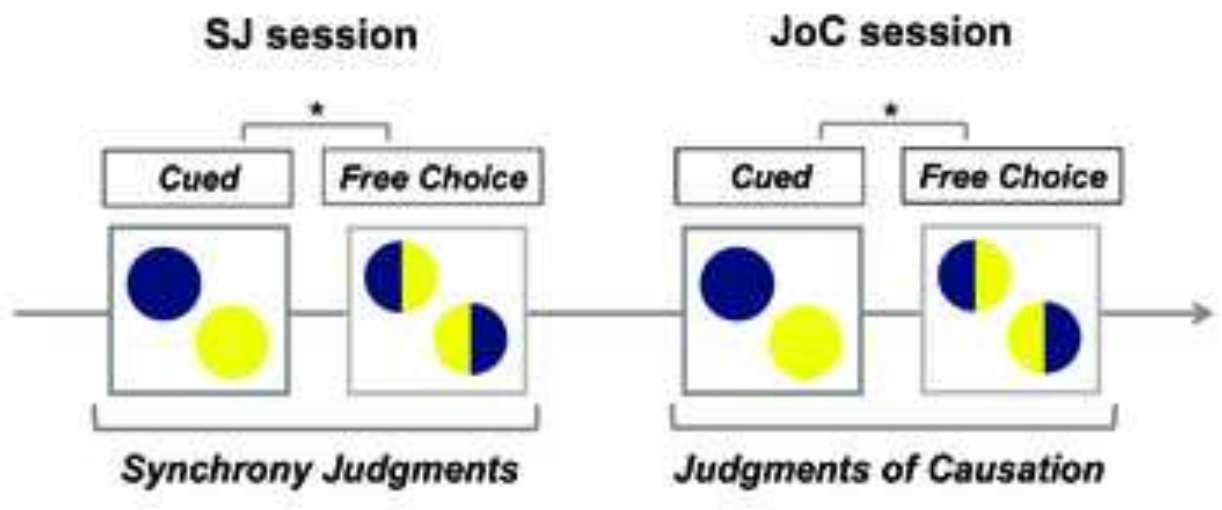

"counterbalanced order

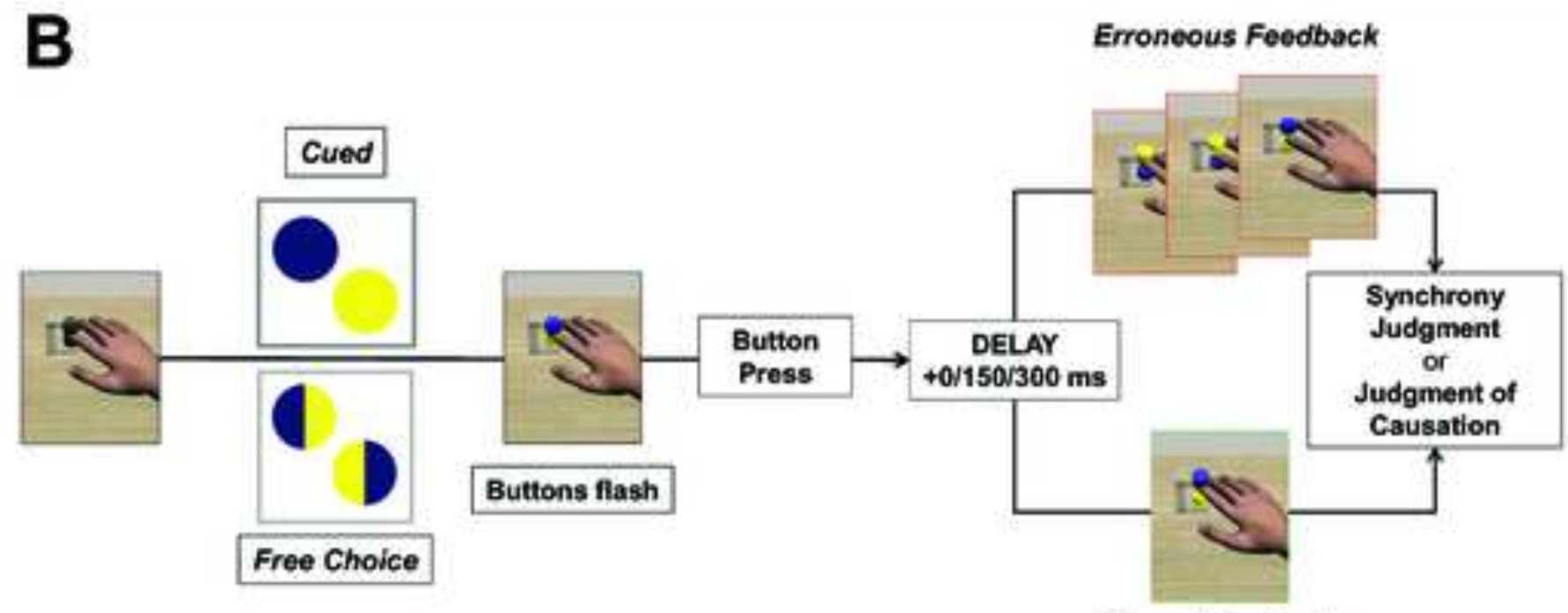

Correct feedback

C

Initial disposition

Congruent Movement,

Achieved Goal
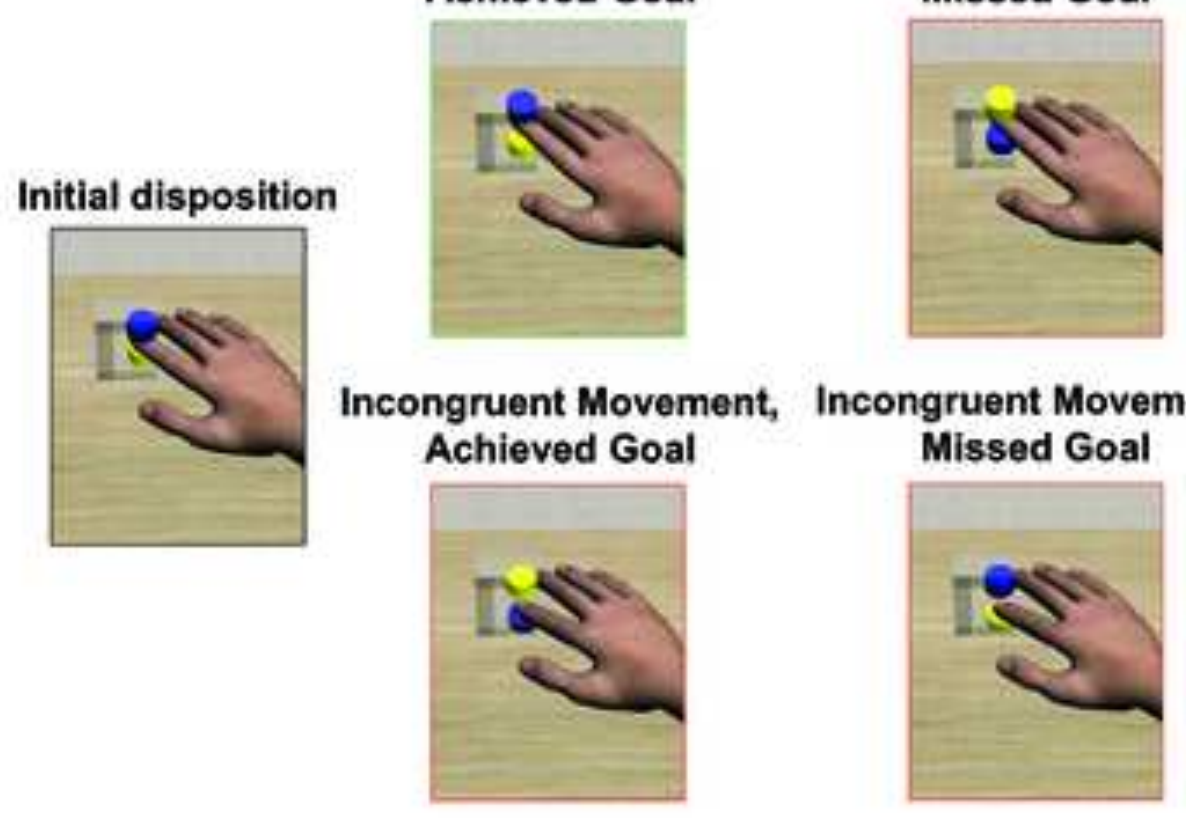

Congruent Movement,

Missed Goal

Real Errors

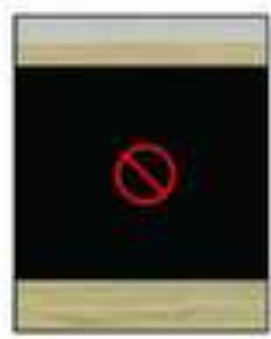




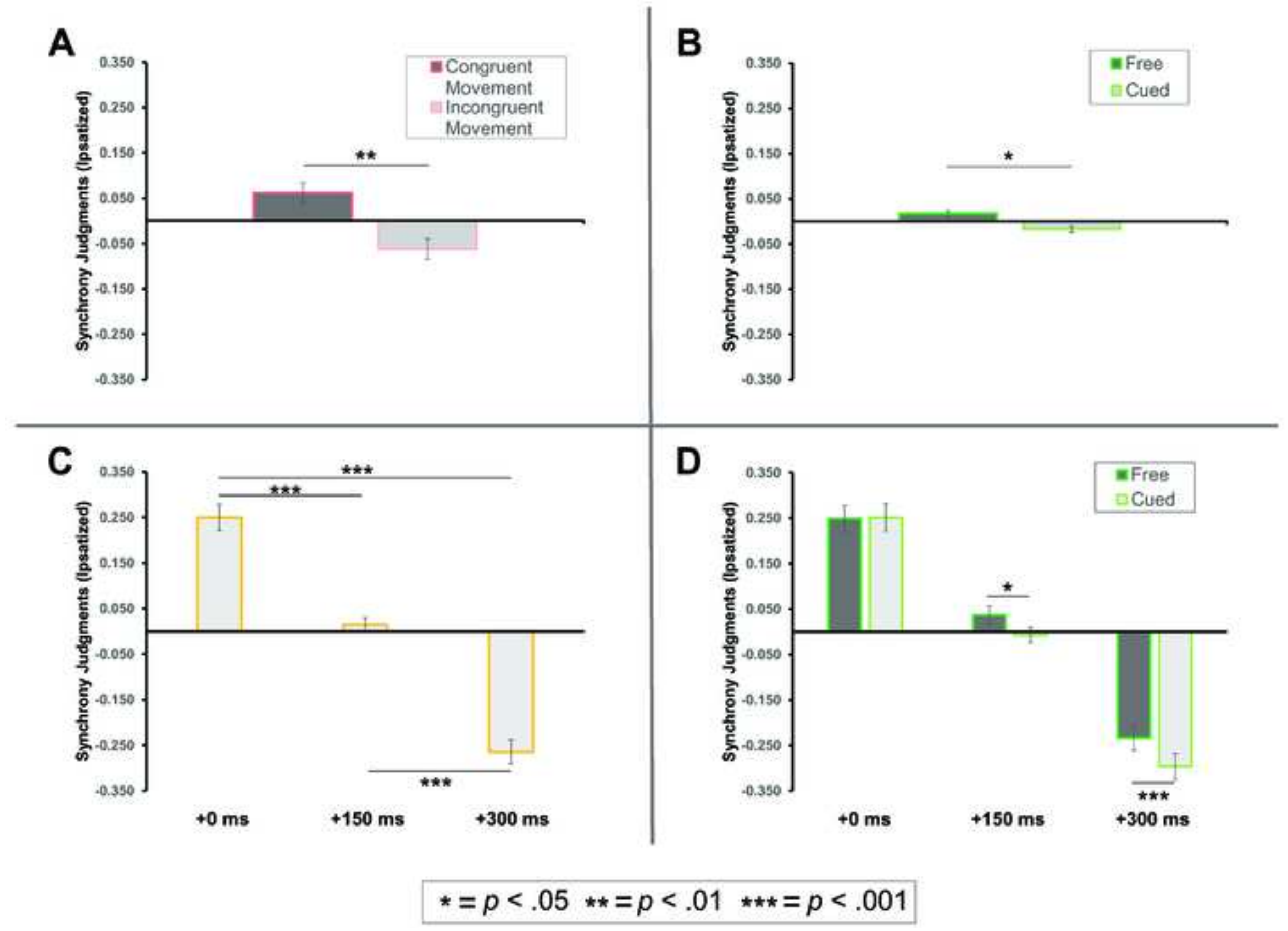

$$
\star=p<.05 * \star=p<.01 \star \star \star *=p<.001
$$




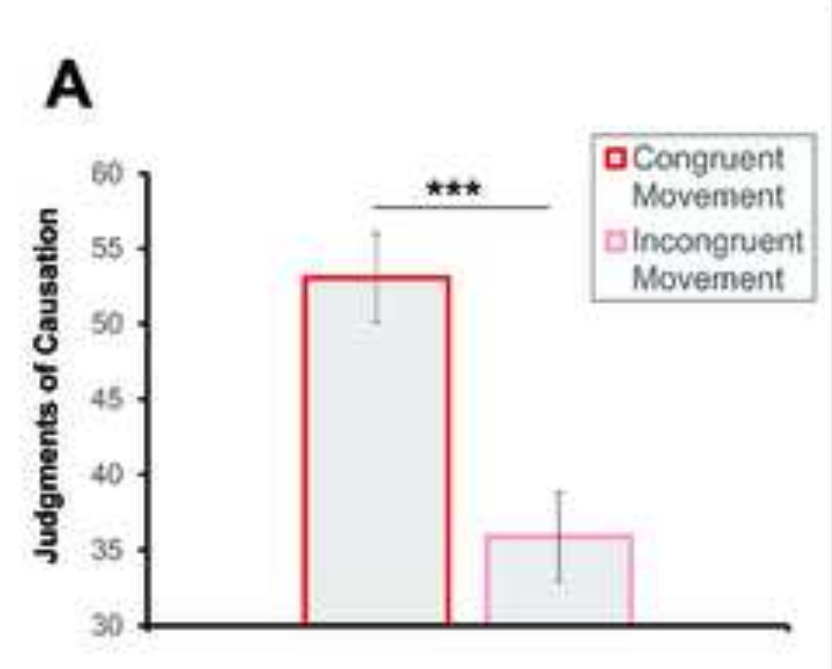

B

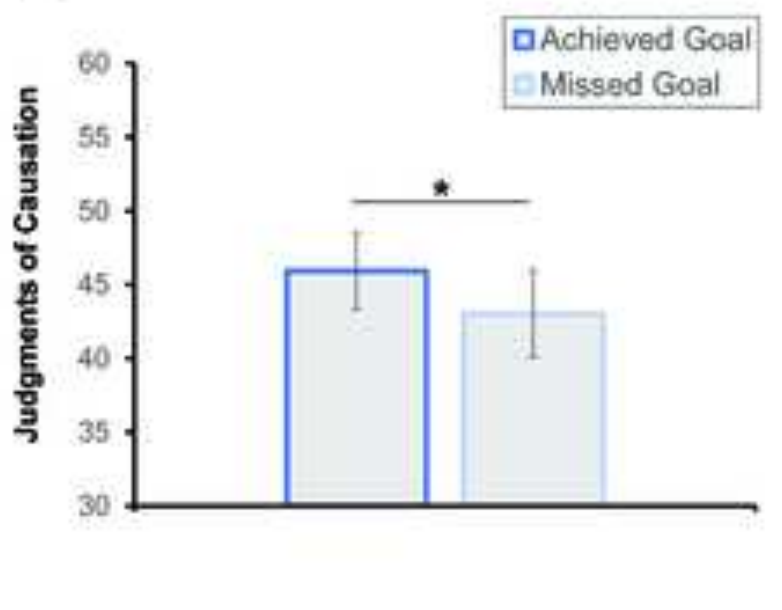

C

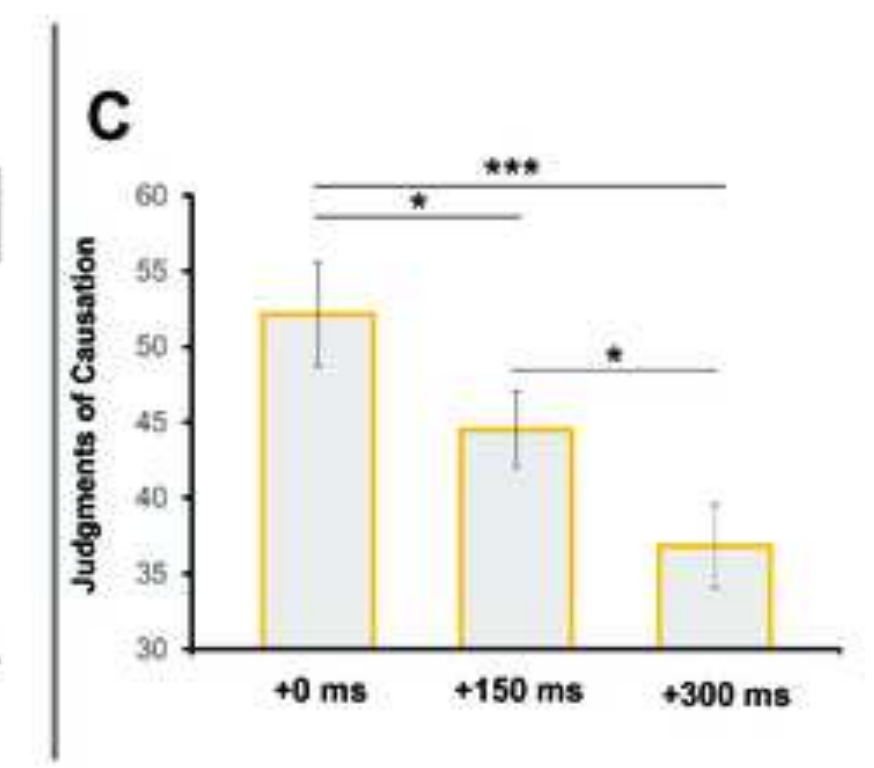

$$
*=p<.05 * * *=p<.001
$$



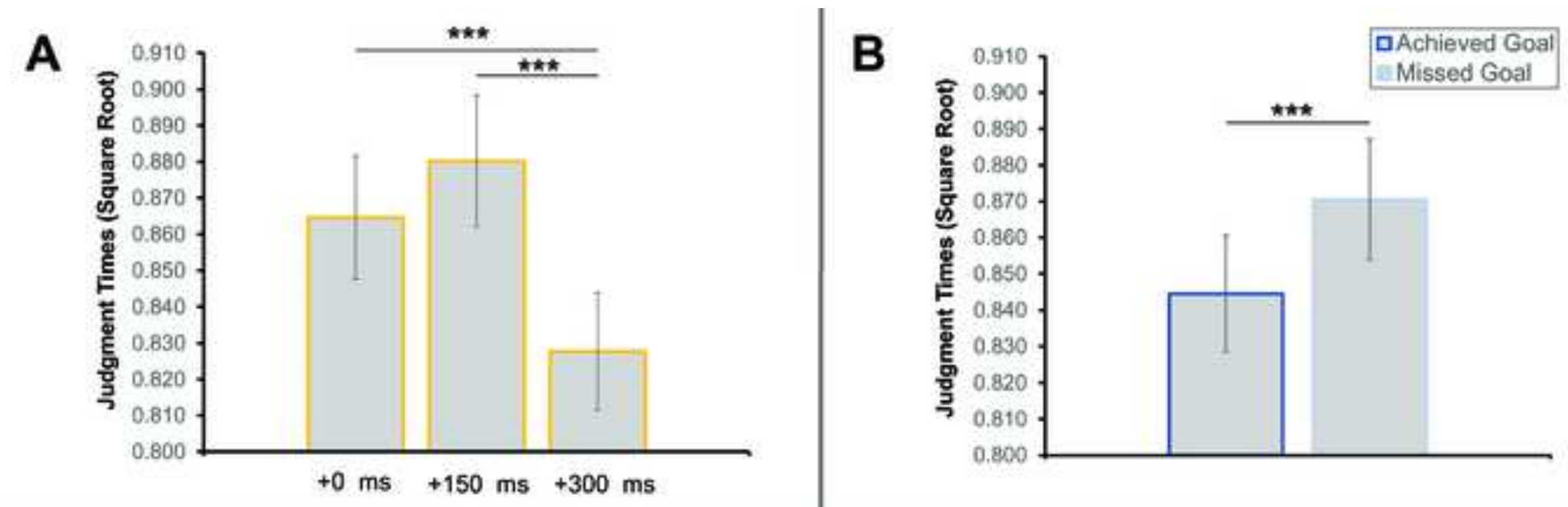

\section{C}

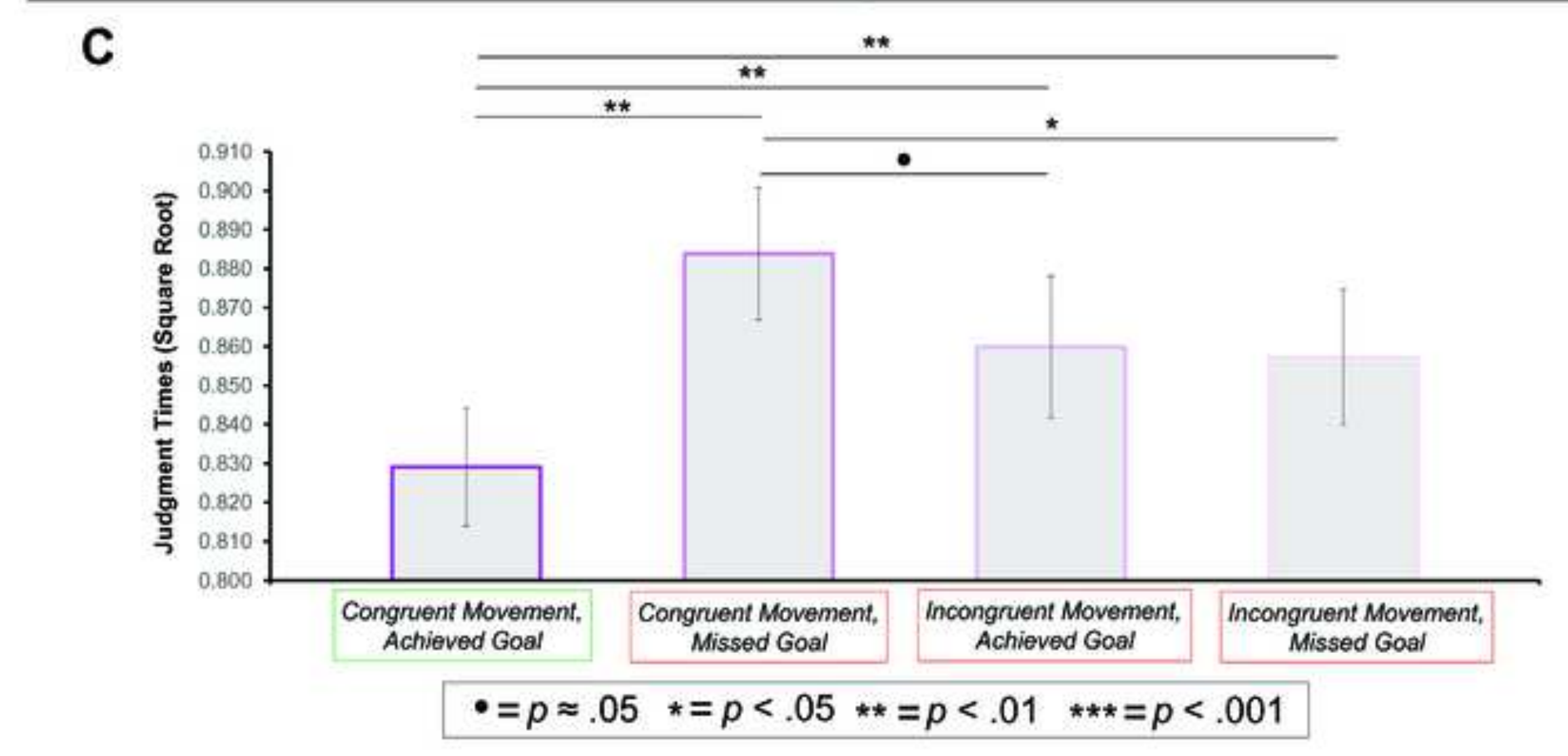

Fire 5 
A

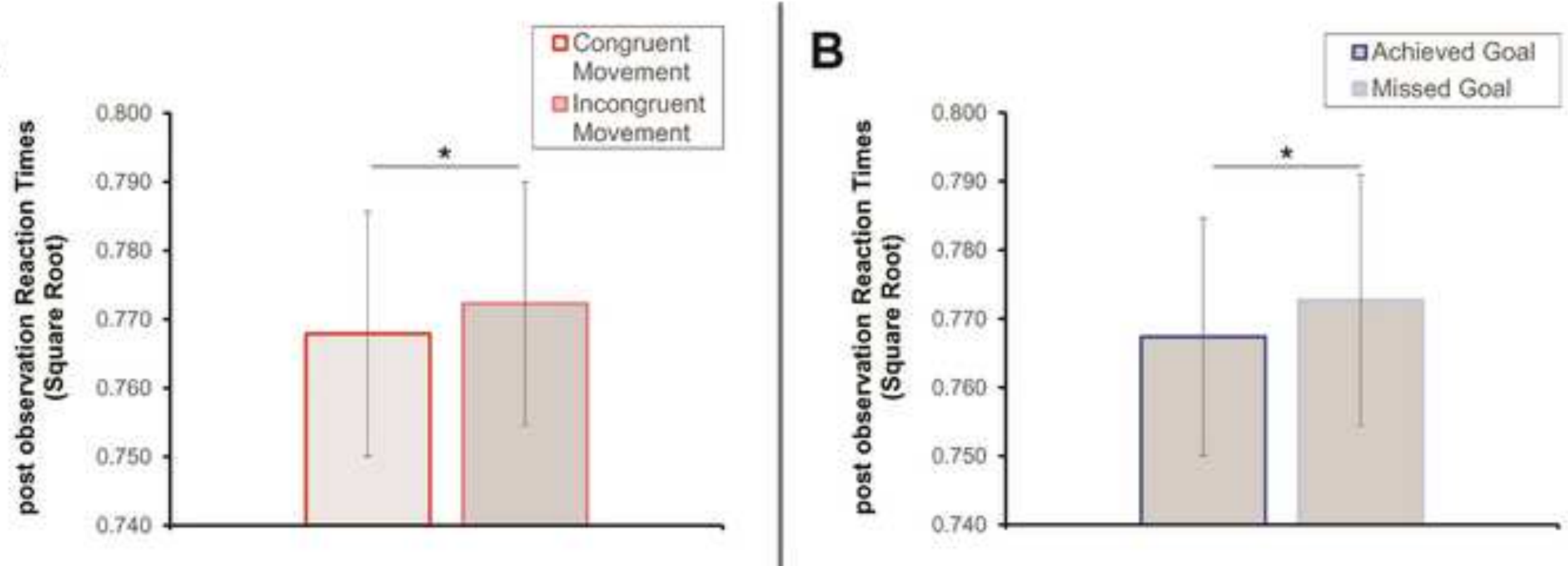


$+0 \mathrm{~ms}$

$+150 \mathrm{~ms}$

$0.251 \pm 0.030$

Cued
$0.249 \pm 0.029$

$+300 \mathrm{~ms}$

\section{Free}

